Hokkaido Mathematical Journal Vol. 45 (2016) p. 1-42

\title{
Dimer models and crepant resolutions
}

\author{
Akira IsHII and Kazushi UeDA \\ (Received July 9, 2013; Revised September 15, 2013)
}

\begin{abstract}
We study variations of tautological bundles on moduli spaces of representations of quivers with relations associated with dimer models under a change of stability parameters. We prove that if the tautological bundle induces a derived equivalence for some stability parameter, then the same holds for any generic stability parameter, and any projective crepant resolution can be obtained as the moduli space for some stability parameter. This result is used in [IU] to prove the abelian McKay correspondence without using the result of Bridgeland, King and Reid [BKR01].
\end{abstract}

Key words: dimer model, toric Calabi-Yau 3-fold, variation of GIT.

\section{Introduction}

In this paper, we study

- variations of geometric invariant theory quotients,

- tautological constructions on moduli spaces, and

- moduli interpretations of toric varieties

for smooth toric Calabi-Yau 3-folds.

The theory of variations of geometric invariant theory quotients of a normal variety [DH98], [Tha96] deals with the dependence of geometric invariant theory quotients on the choice of stability parameters (i.e. the choice of a $G$-linearization on an ample line bundle). The space of stability parameters is divided into chambers by real codimension one walls, and the quotient changes as one moves from one chamber to another. If the generic point is stable for any stability parameter, then different quotients are related by birational transformations such as blow-ups and flips. A prototypical example is a toric variety constructed as the quotient of an affine space by an action of a torus.

Tautological constructions on moduli spaces are fundamental tools to study geometry of moduli spaces. The moduli space that we study in this

2010 Mathematics Subject Classification : 14D20, 14D21. 
paper is the moduli space $\mathcal{M}_{\theta}$ of stable representations of a quiver $\Gamma$ with relations, which is constructed by King [Kin94] using geometric invariant theory. Here $\theta$ is a stability parameter, and we write the tautological bundle on $\mathcal{M}_{\theta}$ as $\mathcal{E}=\bigoplus_{v} \mathcal{L}_{v}$ where $v$ runs over the set of vertices of the quiver. The tautological bundle exists if the dimension vector is a primitive vector [Kin94, Proposition 5.3]. The moduli space $\mathcal{M}_{\theta}$ is an open subscheme of the coarse moduli scheme $\overline{\mathcal{M}_{\theta}}$ parametrizing S-equivalence classes of $\theta$ semistable representations, which is projective over the affine scheme $\overline{\mathcal{M}_{0}}$ defined for the stability parameter 0 . We consider representations with dimension vector $(1, \ldots, 1)$, so that each $\mathcal{L}_{v}$ is a line bundle. The stability parameter $\theta$ is said to be generic if every semistable object is stable. Genericity of $\theta$ means that $\mathcal{M}_{\theta}=\overline{\mathcal{M}_{\theta}}$.

Assume that $\mathcal{M}_{\theta}$ is smooth, and consider the following two conditions:

(T) The tautological bundle $\mathcal{E}$ on the moduli space $\mathcal{M}_{\theta}$ is a tilting object.

(E) The universal morphism $\mathbb{C} \Gamma \rightarrow$ End $\mathcal{E}$ is an isomorphism.

According to Morita theory for derived categories [Bon89], [Ric89], the conditions $(\mathbf{T})+(\mathbf{E})$ imply that the functor

$$
\Phi(-)=\mathbb{R} \Gamma(\mathcal{E} \otimes-): D^{b} \operatorname{coh} \mathcal{M}_{\theta} \rightarrow D^{b} \bmod \mathbb{C} \Gamma
$$

is an equivalence of triangulated categories.

In this paper, we study the special case where the quiver $\Gamma$ with relations comes from a dimer model. A dimer model is a bicolored graph on a real 2-torus, which encodes the information of a quiver with relations. See e.g. [Ken07] and references therein for backgrounds from string theory, and the introduction of [IU] for some of the subsequent development in mathematics. If the dimer model is non-degenerate, then the moduli space $\mathcal{M}_{\theta}$ of stable representations of the corresponding quiver with dimension vector $(1, \ldots, 1)$ with respect to a generic stability parameter $\theta$ is a smooth toric Calabi-Yau 3 -fold, which is a crepant resolution of a Gorenstein affine toric variety $X$ [IU08].

The main result in this paper is the following:

Theorem 1.1 Let $G$ be a non-degenerate dimer model. If the conditions $(\mathbf{T})+(\mathbf{E})$ hold for some generic stability parameter $\theta$, then the same conditions hold for any generic stability parameter. 
Although Theorem 1.1 follows from the result of Bridgeland, King and Reid [BKR01], [vdB04], we give an alternative proof based on an explicit description of variation of moduli spaces under a change of stability parameters. Such a description for moduli spaces of the McKay quiver associated with a finite small abelian subgroup $A$ of $S L_{3}(\mathbb{C})$ is given in [CI04] using the result of Bridgeland, King and Reid. Our proof of Theorem 1.1 works for any non-degenerate dimer models, and does not rely on the result of Bridgeland, King and Reid.

The proof of Theorem 1.1 also gives a generalization of the main result of [CI04] to non-degenerate dimer models:

Corollary 1.2 Let $G$ be a non-degenerate dimer model satisfying conditions $(\mathbf{T})+(\mathbf{E})$ for some (and hence any) generic stability parameter. Then any projective crepant resolution of the Gorenstein affine toric variety $X$ associated with the dimer model $G$ is obtained as the moduli space $\mathcal{M}_{\theta}$ for some generic stability parameter $\theta$.

This leads to a fine moduli interpretation of any projective crepant resolution of any 3 -dimensional Gorenstein affine toric variety $X$. Indeed, every such variety $X$ is determined by a lattice polygon $\Delta$, and [IU, Corollary 1.2] provides a consistent dimer model $G$ wose characteristic polygon coincides with $\Delta$. Then [IU, Theorem 1.4] shows that conditions $(\mathbf{T})+(\mathbf{E})$ hold for any generic stability condition $\theta$, and the claim follows from Corollary 1.2.

Theorem 1.1 is used in [IU] to circumvent the use of the result of Bridgeland, King and Reid [BKR01] in the proof of the main result. Since the main result of [IU] contains the abelian McKay correspondence as a special case, this gives a new proof of the abelian McKay correspondence as a derived equivalence. This paper is separated from [IU] since the technique used in this paper is different from the main line of the argument in [IU], and only the statement of Theorem 1.1 is needed in [IU]. Many of the arguments in this paper are parallel to those in [CI04]; the main difference is that our argument works for general dimer models and does not depend on the result of Bridgeland, King and Reid [BKR01].

This paper is organized as follows: In Section 2, we recall basic definitions on dimer models and associated quivers. In Section 3, we recall the definition of the moduli space of stable representations of quivers. In Section 4, we recall the construction of the moduli space due to King [Kin94]. It is based on geometric invariant theory, and resulting moduli space comes 
naturally with an ample line bundle. In Section 5, we recall the main result of [IU08] which deals with the case when the quiver with relations comes from a non-degenerate dimer model. In Section 6, we describe walls and chambers in the space of stability parameters. In Section 7 , we recall the definition of a tilting object and its relation with the derived equivalence. In Section 8, we give a natural identification between the space of stability parameters and a subspace of the Grothendieck group of finitely-generated modules over the path algebra of the quiver. In Section 9, we assume the derived equivalence and show that a primitive contraction of the moduli space occurs on the walls in the space of stability parameters. In Section 10, we study destabilizing sequences. In Section 11, we study variation of tautological bundles and prove Theorem 1.1. In Section 12, we give the proof of Corollary 1.2.

\section{Dimer models and quivers}

Let $T=\mathbb{R}^{2} / \mathbb{Z}^{2}$ be a real two-torus equipped with an orientation. A bicolored graph on $T$ consists of

- a finite set $B \subset T$ of black nodes,

- a finite set $W \subset T$ of white nodes, and

- a finite set $E$ of edges, consisting of embedded closed intervals $e$ on $T$ such that one boundary of $e$ belongs to $B$ and the other boundary belongs to $W$. We assume that two edges intersect only at the boundaries.

A face of a graph is a connected component of $T \backslash \cup_{e \in E} e$. A bicolored graph $G$ on $T$ is called a dimer model if every face is simply-connected and there is no univalent node.

A quiver consists of

- a set $V$ of vertices,

- a set $A$ of arrows, and

- two maps $s, t: A \rightarrow V$ from $A$ to $V$.

For an arrow $a \in A$, the vertices $s(a)$ and $t(a)$ are said to be the source and the target of a respectively. A path on a quiver is an ordered set of arrows $\left(a_{n}, a_{n-1}, \ldots, a_{1}\right)$ such that $s\left(a_{i+1}\right)=t\left(a_{i}\right)$ for $i=1, \ldots, n-1$. We also allow for a path of length zero, starting and ending at the same vertex. The path algebra $\mathbb{C} Q$ of a quiver $Q=(V, A, s, t)$ is the algebra spanned by 
the set of paths as a vector space, and the multiplication is defined by the concatenation of paths;

$$
\left(b_{m}, \ldots, b_{1}\right) \cdot\left(a_{n}, \ldots, a_{1}\right)= \begin{cases}\left(b_{m}, \ldots, b_{1}, a_{n}, \ldots, a_{1}\right) & s\left(b_{1}\right)=t\left(a_{n}\right) \\ 0 & \text { otherwise }\end{cases}
$$

Paths of length zero correspond to idempotents in the path algebra. A quiver with relations is a pair of a quiver and a two-sided ideal $\mathcal{I}$ of its path algebra. For a quiver $\Gamma=(Q, \mathcal{I})$ with relations, its path algebra $\mathbb{C} \Gamma$ is defined as the quotient algebra $\mathbb{C} Q / \mathcal{I}$. Two paths $a$ and $b$ are said to be equivalent if they give the same element in $\mathbb{C} \Gamma$.

A dimer model $(B, W, E)$ encodes the information of a quiver $\Gamma=$ $(V, A, s, t, \mathcal{I})$ with relations in the following way: The set $V$ of vertices is the set of faces and the set $A$ of arrows is the set $E$ of edges of the graph. The directions of the arrows are determined by the colors of the nodes of the graph, so that the white node $w \in W$ is on the right of the arrow. One can find an example in Figure 12.1 below.

The relations of the quiver are described as follows: For an arrow $a \in A$, there exist two paths $p_{+}(a)$ and $p_{-}(a)$ from $t(a)$ to $s(a)$, the former going around the white node connected to $a \in E=A$ clockwise and the latter going around the black node connected to $a$ counterclockwise. Then the ideal $\mathcal{I}$ of the path algebra is generated by $p_{+}(a)-p_{-}(a)$ for all $a \in A$.

A small cycle on a quiver coming from a dimer model is the product of arrows surrounding only a single node of the dimer model. Small cycles starting from a fixed vertex are equivalent to each other. Hence the sum $\omega$ of small cycles over the set of vertices is a well-defined element of the path algebra, which belongs to the center of the path algebra.

A perfect matching (or a dimer configuration) on a bicolored graph $G=$ $(B, W, E)$ is a subset $D$ of $E$ such that for any node $v \in B \cup W$, there is a unique edge $e \in D$ connected to $v$.

A dimer model is said to be non-degenerate if for any edge $e \in E$, there is a perfect matching $D$ such that $e \in D$.

\section{Moduli spaces of quiver representations}

A representation of a quiver $\Gamma=(V, A, s, t, \mathcal{I})$ with relations is a module over the path algebra $\mathbb{C} \Gamma$. In other words, a representation of $\Gamma$ is a collection 
$M=\left(\left(M_{v}\right)_{v \in V},\left(\psi_{a}\right)_{a \in A}\right)$ of vector spaces $M_{v}$ for $v \in V$ and linear maps $\psi_{a}: M_{s(a)} \rightarrow M_{t(a)}$ for $a \in A$ satisfying relations in $\mathcal{I}$.

The dimension vector of a finite-dimensional representation $\left(\left(M_{v}\right)_{v \in V},\left(\psi_{a}\right)_{a \in A}\right)$ is defined as $\left(\operatorname{dim} M_{v}\right)_{v \in V} \in \mathbb{Z}^{V}$. The support of a representation is the set of vertices $v \in V \operatorname{such}$ that $\operatorname{dim} M_{v} \neq 0$.

A stability parameter $\theta$ for a fixed dimension vector $d$ is an element of

$$
\Theta=\left\{\theta \in \operatorname{Hom}\left(\mathbb{Z}^{V}, \mathbb{Z}\right) \mid \theta(d)=0\right\} .
$$

A $\mathbb{C} \Gamma$-module $M$ with dimension vector $d$ is said to be $\theta$-stable if for any non-trivial submodule $0 \neq N \subsetneq M$, one has $\theta(\operatorname{dim} N)>\theta(\operatorname{dim} M)=0$. The module $M$ is $\theta$-semistable if $\theta(\operatorname{dim} N) \geq \theta(\operatorname{dim} M)$ holds instead of $\theta(\operatorname{dim} N)>\theta(\operatorname{dim} M)$. A stability parameter $\theta$ is said to be generic with respect to a fixed dimension vector if semistability implies stability. A pair $(\theta, \eta)$ of stability parameters are said to define the same stability condition with respect to $d$ if a $\mathbb{C} \Gamma$-module $M$ with dimension vector $d$ is $\theta$-stable if and only if it is $\eta$-stable. This stability condition is introduced by King [Kin94] to construct the moduli space $\mathcal{M}_{\theta}$ representing (the sheafification of) the functor

$$
\begin{aligned}
& (\mathcal{S} \operatorname{ch} / \mathbb{C}) \rightarrow \\
& \Psi \quad \Psi \\
& T \mapsto \quad \mapsto \text { flat families over } T \text { of } \theta \text {-stable representations of } \Gamma \text { \} } / \sim
\end{aligned}
$$

from the category $(\mathcal{S}$ ch $/ \mathbb{C})$ of schemes of finite type over $\mathbb{C}$ to the category $(\mathcal{S} e t)$ of sets. Here, a flat family of representations of $\Gamma$ over $T$ consists of a collection $\left(\mathcal{L}_{v}\right)_{v \in V}$ of vector bundles on $T$ for each vertex $v$ of $\Gamma$ and a collection $\left(\phi_{a}\right)_{a \in A}$ of morphisms $\phi_{a}: \mathcal{L}_{s(a)} \rightarrow \mathcal{L}_{t(a)}$ for each arrow $a$ of $\Gamma$ satisfying the relations $\mathcal{I}$ of $\Gamma$. Two families $\left(\left(\mathcal{L}_{v}\right)_{v \in V},\left(\phi_{a}\right)_{a \in A}\right)$ and $\left(\left(\mathcal{L}_{v}^{\prime}\right)_{v \in V},\left(\phi_{a}^{\prime}\right)_{a \in A}\right)$ are equivalent if there are a line bundle $\mathcal{L}$ and a collection $\left(\psi_{v}\right)_{v \in V}$ of isomorphisms $\psi_{v}: \mathcal{L}_{v} \stackrel{\sim}{\longrightarrow} \mathcal{L}_{v}^{\prime} \otimes \mathcal{L}$ of vector bundles such that the diagram

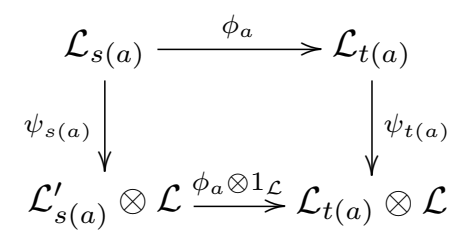


commutes for all $a \in A$. In our case, the dimension vector $(1, \ldots, 1)$ is primitive, so that we do not have to sheafify the functor, and there is a universal family over the moduli space. The bundles $\mathcal{L}_{v}$ in the universal family are called the tautological bundles. We have a freedom of simultaneously tensoring a line bundle in the choice of the tautological bundles. In this paper, we choose a vertex $v_{0}$ of the quiver $\Gamma$ once and for all, and normalize the tautological bundle so that $\mathcal{L}_{v_{0}}$ is the trivial bundle. The moduli space of $\theta$-stable $\mathbb{C} \Gamma$-modules with dimension vector $(1, \ldots, 1)$ is denoted by $\mathcal{M}_{\theta}$. On the other hand, the moduli space $\overline{\mathcal{M}}_{\theta}$ of $\theta$-semistable modules does not represent the moduli functor, but parametrizes S-equivalence classes of $\theta$-semistable modules.

\section{The ample line bundle on the moduli space}

The construction of the moduli spaces $\mathcal{M}_{\theta}$ and $\overline{\mathcal{M}}_{\theta}$ by King [Kin94] is based on geometric invariant theory, and comes with a natural ample line bundle. Here we recall the construction in the case where the dimension vector is $(1, \ldots, 1)$. Fix one-dimensional $\mathbb{C}$-vector spaces $M_{v}=\mathbb{C}$ for each vertex $v \in V$ and consider the affine scheme

$$
\mathcal{N}=\left\{\left(\psi_{a}\right)_{a \in A} \in \mathbb{C}^{A} \mid\left(\psi_{a}\right)_{a \in A} \text { satisfies the relations in } \mathcal{I}\right\} .
$$

Let

$$
\mathcal{G}=\left(\mathbb{C}^{\times}\right)^{V}
$$

be the group of gauge transformations, which acts on $\mathcal{N}$ by

$$
\left(g_{v}\right)_{v \in V}:\left(\psi_{a}\right)_{a \in A} \mapsto\left(\psi_{a}^{\prime}=g_{t(a)} \cdot \psi_{a} \cdot g_{s(a)}^{-1}\right)_{a \in A}
$$

The action of the diagonal subgroup $\mathbb{C}^{\times} \subset \mathcal{G}$ is trivial, and the quotient group will be denoted by $\mathbb{P} \mathcal{G}=\mathcal{G} / \mathbb{C}^{\times}$. A stability parameter $\theta \in \Theta$ defines the character

$$
\begin{array}{cccc}
\tilde{\chi}: & \mathcal{G} & \rightarrow & \mathbb{C}^{\times} \\
\Psi & & \Psi \\
\left(g_{v}\right)_{v \in V} & \mapsto & \prod_{v \in V} g_{v}^{\theta(v)},
\end{array}
$$


which descends to the character $\chi: \mathbb{P G} \rightarrow \mathbb{C}^{\times}$by the condition $\theta(1, \ldots, 1)=$ 0 . Let $\mathbb{C}[\mathcal{N}]_{\chi^{n}}^{\mathcal{G}}$ be the space of semi-invariant functions of weight $\chi^{n}$ on $\mathcal{N}$, and consider the graded ring $R_{\chi}=\bigoplus_{n=0}^{\infty} \mathbb{C}[\mathcal{N}]_{\chi^{n}}^{\mathcal{G}}$.

The moduli space $\overline{\mathcal{M}}_{\theta}$ is obtained as $\overline{\mathcal{M}}_{\theta}=\operatorname{Proj} R_{\chi}$, and the moduli space $\mathcal{M}_{\theta}$ is its open subscheme parametrizing stable orbits. It follows from the construction that the moduli space $\mathcal{M}_{\theta}$ comes with an ample line bundle $\mathcal{O}_{\mathcal{M}_{\theta}}(1)$.

A choice of a splitting $s: \mathbb{P G} \rightarrow \mathcal{G}$ defines tautological bundles $\mathcal{L}_{v}$ for $v \in V$ as the line bundle associated with the character $\pi_{v} \circ s: \mathbb{P G} \rightarrow \mathbb{C}^{\times}$ of $\mathbb{P G}$, where $\pi_{v}: \mathcal{G} \rightarrow \mathbb{C}^{\times}$is the projection $\left(g_{w}\right)_{w \in V} \mapsto g_{v}$ to the $v$ th component. Our choice for the splitting $s$ is such that $s(\alpha)_{v_{0}}=1$ for the fixed vertex $v_{0}$ and any $\alpha \in \mathbb{P} \mathcal{G}$. This gives the normalization of the tautological bundles such that $\mathcal{L}_{v_{0}}$ is the trivial bundle.

Let $L_{\theta}: \Theta \rightarrow$ Pic $\mathcal{M}_{\theta}$ be the map defined by

$$
\begin{aligned}
& L_{\theta}: \Theta \rightarrow \quad \operatorname{Pic} \mathcal{M}_{\theta} \\
& \Psi \quad \Psi \\
& \eta \mapsto \bigotimes_{v \in V} \mathcal{L}_{v}^{\otimes \eta(v)},
\end{aligned}
$$

which we extend to the map $L_{\theta} \otimes \operatorname{id}_{\mathbb{Q}}: \Theta_{\mathbb{Q}} \rightarrow \operatorname{Pic}\left(\mathcal{M}_{\theta}\right)_{\mathbb{Q}}$ where the subscript denotes the tensor product with $\mathbb{Q}$. It follows from the definition that

$$
L_{\theta}(\theta)=\mathcal{O}_{\mathcal{M}_{\theta}}(1) .
$$

The definition of stability condition makes sense also for $\theta \in \Theta_{\mathbb{Q}}=\Theta \otimes \mathbb{Q}$ or $\Theta_{\mathbb{R}}=\Theta \otimes \mathbb{R}$. For any $\theta \in \Theta_{\mathbb{R}}$, one can find a stability parameter $\theta^{\prime} \in \Theta$ defining the same stability condition as $\theta$. Then the moduli space $\mathcal{M}_{\theta^{\prime}}$ is isomorphic to $\mathcal{M}_{\theta}$, and the tautological bundle is the $\mathbb{R}$-line bundle defined as $L_{\theta^{\prime}}(\theta) \in \operatorname{Pic}\left(\mathcal{M}_{\theta}\right)_{\mathbb{R}}$.

\section{Moduli spaces associated with non-degenerate dimer models}

Assume that the quiver $\Gamma$ with relations comes from a dimer model $G$.

Theorem 5.1 ([IU08, Proposition 5.1]) If the dimer model $G$ is nondegenerate, then the moduli space $\mathcal{M}_{\theta}$ is a smooth toric Calabi-Yau 3-fold for generic $\theta$.

The dense torus $\mathbb{T} \subset \mathcal{M}_{\theta}$ is defined as 


$$
\mathbb{T}=\left\{\left[\left(\left(M_{v}\right)_{v \in V},\left(\psi_{a}\right)_{a \in A}\right)\right] \in \mathcal{M}_{\theta} \mid \psi_{a} \neq 0 \text { for any } a \in A\right\},
$$

which acts on $\mathcal{M}_{\theta}$ by pointwise multiplication. A perfect matching $D$ gives a represenation $\psi^{D}$ of $\Gamma$ defined by

$$
\psi_{a}^{D}= \begin{cases}0 & a \in D \\ 1 & \text { otherwise }\end{cases}
$$

A perfect matching is said to be $\theta$-stable if $\psi^{D}$ is a $\theta$-stable representation of $\Gamma$. Any toric divisor in $\mathcal{M}_{\theta}$ is written as

$$
\left\{\left[\left(\left(M_{v}\right)_{v \in V},\left(\psi_{a}\right)_{a \in A}\right)\right] \in \mathcal{M}_{\theta} \mid \psi_{a}=0 \text { for any } a \in D\right\},
$$

for a $\theta$-stable perfect matching $D \subset E$. This correspondence gives a bijection between toric divisors of $\mathcal{M}_{\theta}$ and $\theta$-stable perfect matchings on $G$.

For a not necessarily generic stability parameter $\theta$, the moduli space $\overline{\mathcal{M}}_{\theta}$ still contains $\mathbb{T}$ as an open subset, since any object in $\mathbb{T}$ has no proper subobject, and hence $\theta$-stable for any $\theta$. We write the normalization of the irreducible component of $\overline{\mathcal{M}}_{\theta}$ containing $\mathbb{T}$ as $X_{\theta}$. Theorem 5.1 gives $X_{\theta}=\mathcal{M}_{\theta}$ for generic $\theta$, and $X_{0}$ is the affine toric variety associated with the cone $\mathbb{R} \cdot(\Delta \times\{1\}) \subset \mathbb{R}^{3}$ over a convex lattice polygon $\Delta \subset \mathbb{R}^{2}$ called the characteristic polygon [IU08, Proposition 6.3].

\section{Walls and chambers for stability parameters}

A proper non-empty subset $R \subset V$ defines a wall

$$
W_{R}=\left\{\theta \in \Theta_{\mathbb{R}} \mid \theta\left(\chi_{R}\right)=0\right\}
$$

in the space of stability parameters, where

$$
\chi_{R}(v)= \begin{cases}1 & v \in R \\ 0 & \text { otherwise }\end{cases}
$$

is the characteristic function of $R$.

Lemma 6.1 A stability parameter is generic if it does not lie on any wall $W_{R}$. 
Proof. If there is a strictly $\theta$-semistable object $E$, then there is a subobject $S \subset E$ such that

$$
\theta(\operatorname{dim} S)=0
$$

Since $\operatorname{dim} E=\chi_{V}$, the dimension vector $\operatorname{dim} S$ is the characteristic function of the support $R$ of $S$, and $\theta$ lies on the wall $W_{R}$.

Lemma 6.2 Assume $\Gamma$ comes from a non-degenerate dimer model $G$. Let $\theta$ be a generic stability parameter. Then the set

$$
C=\left\{\begin{array}{l|l}
\eta \in \Theta_{\mathbb{R}} & \begin{array}{c}
\text { For any } \mathbb{C} \Gamma \text {-module } M \text { of dimension vector } \chi_{V}, \\
M \text { is } \theta \text {-stable if and only if } M \text { is } \eta \text {-stable }
\end{array}
\end{array}\right\}
$$

is the convex open rational polyhedral cone consisting of $\eta \in \Theta_{\mathbb{R}}$ such that $\eta(\operatorname{dim} F)>0$ for any non-trivial submodule $F$ of any $\theta$-stable representation $M$.

Proof. Let $C^{\prime}$ be the convex open cone consisting of $\eta \in \Theta_{\mathbb{R}}$ such that $\eta(\operatorname{dim} F)>0$ for any non-trivial submodule $F$ of any $\theta$-stable representation $M$. Although the set of $\theta$-stable representations is infinite, the set of dimension vectors of non-trivial submodules $F$ of a $\theta$-stable representation is finite, so that $C^{\prime}$ is a rational polyhedral cone defined by finitely many inequalities. The inclusion $C \subset C^{\prime}$ is obvious and we show the converse. Assume for a parameter $\eta \in C^{\prime}$, a representation $E$ is $\eta$-stable but not $\theta$-stable. If $\eta^{\prime} \in C^{\prime}$ is close enough to $\eta$, then $E$ is also $\eta^{\prime}$-stable. Replacing $\eta$ by $\eta^{\prime}$, we may assume $\eta$ is generic. Then since both $\mathcal{M}_{\theta}$ and $\mathcal{M}_{\eta}$ are crepant resolutions of the same toric variety by [IU08], the inclusion $\mathcal{M}_{\theta} \subset \mathcal{M}_{\eta}$ implies $\mathcal{M}_{\theta}=\mathcal{M}_{\eta}$, contradicting $[E] \in \mathcal{M}_{\eta} \backslash \mathcal{M}_{\theta}$.

Remark 6.3 The proof of Lemma 6.2 does not show that $C$ coincides with the set

$$
\left\{\begin{array}{l|l}
\eta \in \Theta_{\mathbb{R}} & \begin{array}{c}
\text { For any } \mathbb{C} \Gamma \text {-module } M \text { of dimension vector } \chi_{V} \\
M \text { is } \theta \text {-semistable if and only if } M \text { is } \eta \text {-semistable }
\end{array}
\end{array}\right\} .
$$

The coincidence is equivalent to the genericity of all the parameters in $C$, which follows from the connectedness of $\overline{\mathcal{M}_{\eta}}$ for any $\eta \in C$. Once we establish $(\mathbf{T})+(\mathbf{E})$, the argument of [BKR01, Section 8] shows the connectedness 
of $\overline{\mathcal{M}}_{\eta}$ for any $\eta \in C$.

The open cone $C \subset \Theta$ is called a chamber, which is separated from other chambers by walls. The space $\Theta$ of stability parameters is divided into finitely-many walls and chambers, and any stability parameters in the same chamber give the same stability condition. Since the map $L_{\theta}: \Theta \rightarrow \operatorname{Pic} \mathcal{M}_{\theta}$ defined in (4.1) depends only on the chamber $C$ containing $\theta$, we often write $L_{C}$ instead of $L_{\theta}$.

Let $\left(\theta, \theta^{\prime}\right)$ be a pair of generic stability parameters in a pair $\left(C, C^{\prime}\right)$ of adjacent chambers in $\Theta$ separated by a wall $W_{R}$. We assume that the stability parameter

$$
\theta_{0}=\frac{1}{2}\left(\theta+\theta^{\prime}\right)
$$

is generic on the wall in the sense that $\theta_{0} \in W_{R}$ and it does not lie on any other walls. Recall that $X_{\theta_{0}}$ is the normalization of the distinguished irreducible component of $\overline{\mathcal{M}}_{\theta_{0}}$ containing the dense torus $\mathbb{T} \subset \overline{\mathcal{M}}_{\theta_{0}}$. By the definition of GIT quotient, there is a projective morphism $\mathcal{M}_{\theta} \rightarrow \overline{\mathcal{M}}_{\theta_{0}}$, which factors through $X_{\theta_{0}}$ as $\mathcal{M}_{\theta} \stackrel{f}{\rightarrow} X_{\theta_{0}} \rightarrow \overline{\mathcal{M}}_{\theta_{0}}$ since $\mathcal{M}_{\theta}$ is smooth and irreducible. The morphism $f^{\prime}: \mathcal{M}_{\theta^{\prime}} \rightarrow X_{\theta_{0}}$ is defined similarly, and one obtains the diagram

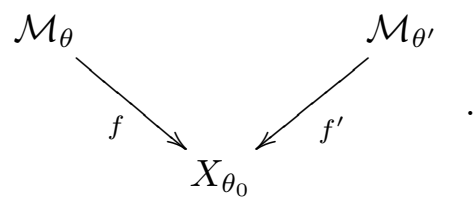

Let $\mathcal{O}_{X_{\theta_{0}}}(1)$ be the pull-back of the ample line bundle $\mathcal{O}_{\overline{\mathcal{M}}_{\theta_{0}}}(1)$ by the normalization map $X_{\theta_{0}} \rightarrow \overline{\mathcal{M}}_{\theta_{0}}$.

Lemma 6.4 The line bundle $L_{C}\left(\theta^{\prime}\right)^{-1}$ is $f$-ample.

Proof. One has $L_{C}\left(\theta_{0}\right) \cong f^{*} \mathcal{O}_{X_{\theta_{0}}}(1)$, so that $L_{C}(\theta) \otimes L_{C}\left(\theta^{\prime}\right) \cong L_{C}\left(2 \theta_{0}\right) \cong$ $f^{*} \mathcal{O}_{X_{\theta_{0}}}(2)$. This shows that $L_{C}\left(\theta^{\prime}\right)^{-1} \cong L_{C}(\theta) \otimes f^{*} \mathcal{O}_{X_{\theta_{0}}}(-2)$ is $f$-ample.

Lemma 6.5 Both $f$ and $f^{\prime}$ are $\mathbb{T}$-equivariant, so that (6.2) is a diagram in the category of toric varieties. 
Proof. This is clear from the definition of $\mathbb{T}$ and its action on $\mathcal{M}_{\theta}, \mathcal{M}_{\theta^{\prime}}$ and $X_{\theta_{0}}$.

\section{Tilting object and derived equivalence}

Definition 7.1 A vector bundle $\mathcal{E}$ on a smooth quasi-projective variety $X$ is acyclic if $\operatorname{Ext}^{k}(\mathcal{E}, \mathcal{E})=0$ for any $k \neq 0$. An acyclic bundle is tilting if the smallest full triangulated subcategory of $D^{b} \operatorname{coh} X$ containing $\mathcal{E}$ and closed under isomorphisms and direct summands is the whole of $D^{b} \operatorname{coh} X$.

A tilting bundle induces a derived equivalence:

Theorem 7.2 (Bondal [Bon89], Rickard [Ric89]) Let $\mathcal{E}$ be a tilting bundle on a smooth quasi-projective variety $X$. Then the functor

$$
\Phi(-)=\mathbb{R} \Gamma(\mathcal{E} \otimes-): D^{b} \operatorname{coh} X \rightarrow D^{b} \bmod A
$$

is an equivalence from $D^{b} \operatorname{coh} X$ to the derived category $D^{b} \bmod A$ of finitelygenerated left modules over the endomorphism algebra $A=\operatorname{Hom}(\mathcal{E}, \mathcal{E})$.

A vector bundle $\mathcal{E}$ on a smooth quasi-projective variety $X$ is a generator of $D^{b} \operatorname{coh} X$ if for any object $\mathcal{F}$ of $D^{b} \operatorname{coh} X$, the condition that $\operatorname{Ext}^{k}(\mathcal{E}, \mathcal{F})=$ 0 for all $k \in \mathbb{Z}$ implies $\mathcal{F} \cong 0$. A vector bundle $\mathcal{E}$ on a smooth quasiprojective variety is a tilting object if and only if it is an acyclic generator (cf. e.g. [HVdB07, Section 7] or [TU10, Section 3]).

It is more common to use the functor

$$
\Psi(-)=\mathbb{R} \operatorname{Hom}(\mathcal{E},-): D^{b} \operatorname{coh} X \rightarrow D^{b} \bmod B
$$

where $B=A^{\text {op }}$ is the opposite ring of $A$. Since $\mathcal{E}$ is a tilting object if and only if $\mathcal{E}^{\vee}$ is a tilting object and $B=A^{\text {op }}=\operatorname{End}\left(\mathcal{E}^{\vee}\right)$, the functor $\Phi$ is an equivalence if and only if the functor $\Psi$ is an equivalence. The advantage of the functor $\Phi$ is that if $X=\mathcal{M}_{\theta}$ is the moduli space of stable representations of the quiver $\Gamma$ with relations and $\mathcal{E}=\bigoplus_{v} \mathcal{L}_{v}$ is the tautological bundle, then the functor

$$
\Phi_{\theta}(-)=\mathbb{R} \Gamma(\mathcal{E} \otimes-): D^{b} \operatorname{coh} \mathcal{M}_{\theta} \rightarrow D^{b} \bmod \mathbb{C} \Gamma,
$$

sends

- the structure sheaf $\mathcal{O}_{y}$ of a point $y \in \mathcal{M}_{\theta}$ to the $\mathbb{C} \Gamma$-module $E_{y}$ 
parametrized by that point, that is, $\Phi_{\theta}\left(\mathcal{O}_{y}\right)=E_{y}$, and

- the line bundle $\mathcal{L}_{v}^{-1}$ to the $\mathbb{C} \Gamma$-module $H^{0}\left(\mathcal{E} \otimes \mathcal{L}_{v}^{-1}\right)$, which is the projective module $P_{v}:=\mathbb{C} \Gamma \cdot e_{v}$ associated with the idempotent $e_{v}=$ $\operatorname{id}_{\mathcal{L}_{v}} \in \mathbb{C} \Gamma \cong \operatorname{End}(\mathcal{E})=\bigoplus_{v, w \in V} H^{0}\left(\mathcal{L}_{v} \otimes \mathcal{L}_{w}^{-1}\right)$, that is, $\Phi_{\theta}\left(\mathcal{L}_{v}^{-1}\right)=$ $P_{v}$.

The full subcategory of $\bmod \mathbb{C} \Gamma$ consisting of finite-dimensional representations will be denoted by $\bmod _{c} \mathbb{C} \Gamma$. A $\mathbb{C} \Gamma$-module $M$ is nilpotent if there is a natural number $n$ such that any path with length greater than $n$ acts on $M$ by zero. The full subcategory of $\bmod _{c} \mathbb{C} \Gamma$ consisting of nilpotent representations will be denoted by $\bmod _{0} \mathbb{C} \Gamma$. The functor $\Phi_{\theta}$ induces functors $D^{b} \operatorname{coh}_{c} \mathcal{M}_{\theta} \rightarrow D^{b} \bmod _{c} \mathbb{C} \Gamma$ and $D^{b} \operatorname{coh}_{0} \mathcal{M}_{\theta} \rightarrow D^{b} \bmod _{0} \mathbb{C} \Gamma$, both of which will be written as $\Phi_{\theta}$ by abuse of notation. Here $\operatorname{coh}_{c} \mathcal{M}_{\theta}$ is the full subcategory of $\operatorname{coh} \mathcal{M}_{\theta}$ consisting of compactly supported sheaves, and $\operatorname{coh}_{0} \mathcal{M}_{\theta}$ is its full subcategory consisting of sheaves supported on the fiber of the unique torus fixed point of $X_{0}$ by the natural map $\mathcal{M}_{\theta} \rightarrow X_{0}$.

\section{Stability parameters and Grothendieck groups}

Assume $\Gamma$ comes from a non-degenerate dimer model $G$ and the conditions $(\mathbf{T})+(\mathbf{E})$ hold for some generic stability parameter $\theta$. The Grothendieck groups of $\operatorname{coh} \mathcal{M}_{\theta}, \operatorname{coh}_{c} \mathcal{M}_{\theta}, \operatorname{coh}_{0} \mathcal{M}_{\theta} \bmod \mathbb{C} \Gamma, \bmod _{c} \mathbb{C} \Gamma$ and $\bmod _{0} \mathbb{C} \Gamma$ will be denoted by $K\left(\mathcal{M}_{\theta}\right), K_{c}\left(\mathcal{M}_{\theta}\right), K_{0}\left(\mathcal{M}_{\theta}\right), K(\Gamma), K_{c}(\Gamma)$ and $K_{0}(\Gamma)$ respectively. Since no path of length zero appears in the relations of $\Gamma$, the Grothendieck group $K_{0}(\Gamma)$ is the abelian group freely generated by the classes of simple modules $S_{v}$ supported on vertices $v$ of the quiver. Our assumption implies that the path algebra $\mathbb{C} \Gamma$ has finite global dimension, so that $K(\Gamma)$ is isomorphic to its subgroup $K^{\text {perf }}(\Gamma)$ generated by the classes of projective modules. For each vertex $v \in V$, there is a projective module $P_{v}=\mathbb{C} \Gamma \cdot e_{v}$, where $e_{v} \in \mathbb{C} \Gamma$ is the idempotent associated with $v$. There is a natural pairing

$$
\chi: K^{\text {perf }}(\Gamma) \times K_{c}(\Gamma) \rightarrow \mathbb{Z}, \quad(\alpha, \beta) \mapsto \sum_{i}(-1)^{i} \operatorname{dim} \operatorname{Ext}^{i}(\alpha, \beta)
$$

called the Euler form, and the numerical Grothendieck group $N_{c}(\Gamma)$ is defined as the quotient of $K_{c}(\Gamma)$ by the radical of the Euler form.

Lemma 8.1 Assume that 
- $K(\Gamma)$ and $K_{0}(\Gamma)$ have the same rank,

- $K(\Gamma)$ is torsion-free, and

- $K_{0}(\Gamma) \rightarrow N_{c}(\Gamma)$ is surjective,

then the map $K_{0}(\Gamma) \rightarrow N_{c}(\Gamma)$ is an isomorphism, and $\left(\left[\mathcal{P}_{v}\right]\right)_{v \in V}$ and $\left(\left[S_{v}\right]\right)_{v \in V}$ are dual bases of $K(\Gamma)$ and $N_{c}(\Gamma)$.

Proof. The modules $\left\{P_{v}\right\}$ and $\left\{S_{v}\right\}$ satisfy

$$
\operatorname{Ext}^{i}\left(P_{v}, S_{w}\right)= \begin{cases}\mathbb{C} & v=w \text { and } i=0, \\ 0 & \text { otherwise. }\end{cases}
$$

Since the classes of $S_{v}$ form a free basis of $K_{0}(\Gamma)$, we obtain a surjection from $K(\Gamma)$ to the dual of $K_{0}(\Gamma)$, which must be an isomorphism by our assumptions. In particular, $\left(\left[\mathcal{P}_{v}\right]\right)_{v \in V}$ and $\left(\left[S_{v}\right]\right)_{v \in V}$ are dual bases of $K(\Gamma)$ and $K_{0}(\Gamma)$. Then the map $K_{0}(\Gamma) \rightarrow N_{c}(\Gamma)$ has to be an isomorphism.

Lemma 8.2 The rank of $K(\Gamma) \cong K\left(\mathcal{M}_{\theta}\right)$ coincides with the Euler number of $\mathcal{M}_{\theta}$, which coincides with the rank of $K_{0}(\Gamma) \cong K_{0}\left(\mathcal{M}_{\theta}\right)$.

Proof. Since the rank of the Grothendieck group coincides with that of the Chow group, we may consider Chow groups instead of Grothendieck groups. Then the assertion follows from the argument of [Ful93, Section 5.2], which is sketched below for the readers' convenience.

Let $\Sigma$ be the fan which determines the toric variety $\mathcal{M}_{\theta}$ and let $e$ be the number of 3-dimensional cones of $\Sigma$. We can construct a complete simplicial fan $\widetilde{\Sigma}$ containing $\Sigma$ such that $\widetilde{\Sigma}$ has one extra one-dimensional cone and $n$ extra three-dimensional cones, where $n$ is the number of edges of the characteristic polygon. Then $\widetilde{\Sigma}$ determines a projective toric variety $X(\widetilde{\Sigma})$.

If we take the ordering of the three-dimensional cones of $\widetilde{\Sigma}$ in [Ful93, Section 5.2] so that the last $e$ cones are those of $\Sigma$, then in the filtration $X(\widetilde{\Sigma})=Z_{1} \supset Z_{2} \supset \cdots \supset Z_{n+e}$ by closed subschemes in [Ful93, Section 5.2], the subscheme $Z_{n+1}$ is the fiber of the unique torus fixed point of $X_{0}$ by the resolution $\mathcal{M}_{\theta}$ and $Z_{i} \backslash Z_{i+1}$ is an affine space for $i \geq n+1$. Hence $e$ coincides with the rank of the Chow group of $Z_{n}$, which is equal to the rank of $K_{0}\left(\mathcal{M}_{\theta}\right)$.

If we take the ordering so that the first $e$ cones are those of $\Sigma$, then $Z_{1} \backslash Z_{e+1}$ coincides with $\mathcal{M}_{\theta}$ and $Z_{i} \backslash Z_{i+1}$ is an affine space for $i \leq e$. Then 
$e$ also coincides with the Euler number of $\mathcal{M}_{\theta}$ and the rank of the Chow group of $\mathcal{M}_{\theta}$.

The group $K(\Gamma) \cong K\left(\mathcal{M}_{\theta}\right)$ is torsion-free since $\mathcal{M}_{\theta}$ is a toric variety. There is a $\mathbb{C}^{*}$-action on the representations of $\Gamma$ as in [IU08, Section 5] and the limit of an object in $\bmod _{c} \mathbb{C} \Gamma$ with respect to this action lies in $\bmod _{0} \mathbb{C} \Gamma$. It follows that the map $K_{0}(\Gamma) \rightarrow N_{c}(\Gamma)$ is surjective. This concludes the proof of the following:

Proposition 8.3 The map $K_{0}(\Gamma) \stackrel{\sim}{\longrightarrow} N_{c}(\Gamma)$ is an isomorphism, the Euler form $(8.1)$ is perfect, and $\left(\left[\mathcal{P}_{v}\right]\right)_{v \in V}$ and $\left(\left[S_{v}\right]\right)_{v \in V}$ are dual bases of $K(\Gamma)$ and $N_{c}(\Gamma)$.

The space $\mathbb{Z}^{V}$ of dimension vectors can naturally be identified with the numerical Grothendieck group $N_{c}(\Gamma)$. The dual space $\operatorname{Hom}\left(\mathbb{Z}^{V}, \mathbb{Z}\right)$ is identified with the Grothendieck group $K(\Gamma)$ by the Euler form, and the space

$$
\Theta=\left\{\theta \in \operatorname{Hom}\left(\mathbb{Z}^{V}, \mathbb{Z}\right) \mid \theta\left(\chi_{V}\right)=0\right\}
$$

of stability parameters is identified with its codimension one subspace.

\section{The Grothendieck group and the Picard group}

Let $\theta \in \Theta$ be a generic stability parameter. Assume that the conditions $(\mathbf{T})+(\mathbf{E})$ hold for the stability parameter $\theta$, so that one has a derived equivalence

$$
\Phi_{C}(-)=\mathbb{R} \Gamma(\mathcal{E} \otimes-): D^{b} \operatorname{coh} \mathcal{M}_{\theta} \stackrel{\sim}{\longrightarrow} D^{b} \bmod \mathbb{C} \Gamma,
$$

where we have added the subscript $C$ to indicate the dependence on the chamber $C \subset \Theta$ containing $\theta$. The functor $\Phi_{C}$ induces a functor

$$
\Phi_{C}: D^{b} \operatorname{coh}_{0} \mathcal{M}_{\theta} \stackrel{\sim}{\longrightarrow} D^{b} \bmod _{0} \mathbb{C} \Gamma
$$

which is denoted by the same symbol by abuse of notation.

Let $K\left(\mathcal{M}_{\theta}\right)$ and $K_{0}\left(\mathcal{M}_{\theta}\right)$ be the Grothendieck groups of $D^{b} \operatorname{coh} \mathcal{M}_{\theta}$ and $D^{b} \operatorname{coh}_{0} \mathcal{M}_{\theta}$ respectively. One has a decreasing filtration

$$
K\left(\mathcal{M}_{\theta}\right)_{\mathbb{Q}}=F^{0} \supset F^{1} \supset F^{2} \supset F^{3} \supset F^{4}=0
$$


of $K\left(\mathcal{M}_{\theta}\right)_{\mathbb{Q}}=K\left(\mathcal{M}_{\theta}\right) \otimes \mathbb{Q}$ by codimension of the support, and an increasing filtration

$$
0=F_{-1} \subset F_{0} \subset F_{1} \subset F_{2}=K_{0}\left(\mathcal{M}_{\theta}\right)_{\mathbb{Q}}
$$

of $K_{0}\left(\mathcal{M}_{\theta}\right)_{\mathbb{Q}}=K_{0}\left(\mathcal{M}_{\theta}\right) \otimes \mathbb{Q}$ by dimension of the support. Since the Euler form

$$
\chi: K\left(\mathcal{M}_{\theta}\right) \times K_{0}\left(\mathcal{M}_{\theta}\right) \rightarrow \mathbb{Z}, \quad(\alpha, \beta) \mapsto \sum_{i}(-1)^{i} \operatorname{dim} \operatorname{Ext}^{i}(\alpha, \beta)
$$

is perfect by Proposition 8.3, one has

$$
F^{1}=F_{0}^{\perp}, \quad F^{2}=F_{1}^{\perp}, \quad \text { and } \quad F^{3}=F_{2}^{\perp}=0
$$

with respect to the Euler form [CI04, Proposition 5.1]. The equivalence (9.2) induces an isomorphism $\varphi_{C}: K_{0}\left(\mathcal{M}_{\theta}\right) \rightarrow K_{0}(\Gamma)$, whose adjoint with respect to the pairing (9.3) gives an isomorphism

$$
\varphi_{C}^{*}: K(\Gamma) \rightarrow K\left(\mathcal{M}_{\theta}\right)
$$

The isomorphism (9.4) induces an isomorphism

$$
\varphi_{C}^{*}: \Theta \rightarrow F^{1}
$$

since $\Theta \subset \operatorname{Hom}\left(\mathbb{Z}^{V}, \mathbb{Q}\right) \cong \operatorname{Hom}\left(K_{0}(\Gamma), \mathbb{Q}\right) \cong K(\Gamma) \otimes \mathbb{Q}$ is the kernel of $\chi_{V} \in \mathbb{Z}^{V} \cong K_{0}(\Gamma)$ and $\varphi_{C}^{*}\left(\chi_{V}\right)$ is the class of the structure sheaf of a point. Let

$$
\text { det }: F^{1} / F^{2} \stackrel{\sim}{\longrightarrow} \operatorname{Pic}\left(\mathcal{M}_{\theta}\right)_{\mathbb{Q}}
$$

be the isomorphism sending the class $\left[\mathcal{O}_{D}\right]=[\mathcal{O}]-[\mathcal{O}(-D)]$ of the structure sheaf of a divisor $D$ to $\operatorname{det}\left(\mathcal{O}_{D}\right)^{-1}=\mathcal{O}(-D)$. Note that one has

$$
\varphi_{C}^{*}(\theta)=\varphi_{C}^{*}\left(\sum_{v \in V} \theta(v)\left[\mathcal{P}_{v}\right]\right)=\sum_{v \in V} \theta(v)\left[\mathcal{L}_{v}^{-1}\right]
$$

so that the map $L_{C}$ defined in (4.1) can be expressed as 


$$
L_{C}(\theta)=\operatorname{det}^{-1}\left(\left[\varphi_{C}^{*}(\theta)\right]\right) \in \operatorname{Pic}\left(\mathcal{M}_{\theta}\right)_{\mathbb{Q}}
$$

This gives the commutative diagram

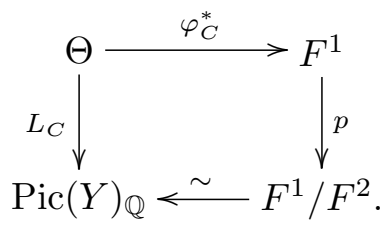

In particular, one has

$$
\operatorname{deg}\left(\left.L_{C}(\theta)\right|_{\ell}\right)=\sum_{v \in V} \chi\left(\mathcal{L}_{v} \otimes \mathcal{O}_{\ell}\right) \theta(v)=\theta\left(\varphi_{C}\left(\mathcal{O}_{\ell}\right)\right)
$$

for a curve $\ell$ on $\mathcal{M}_{\theta}$.

Recall that a contraction is a birational morphism onto a normal variety with a smaller Picard number. A contraction is primitive if it cannot be obtained as the composition of two contractions. According to Wilson [Wil92], the codimension one faces of the ample cone $\operatorname{Amp}(Y)$ of a Calabi-Yau 3-fold correspond to primitive birational contractions, which can be classified into the following three types:

type I: $f$ contracts a curve to a point.

type II: $f$ contracts a surface to a point.

type III: $f$ contracts a surface to a curve.

Lemma 9.1 The morphism $f: \mathcal{M}_{\theta} \rightarrow X_{\theta_{0}}$ is either a primitive contraction or an isomorphism.

Proof. It follows from the definitions of $X_{\theta_{0}}$ and the morphism $\mathcal{M}_{\theta} \rightarrow X_{\theta_{0}}$ that $L_{C}\left(\theta_{0}\right)=f^{*} \mathcal{O}_{X_{\theta_{0}}}(1)$. If $L_{C}\left(\theta_{0}\right)$ is in the interior of the ample cone, then $f$ is an isomorphism. If $L_{C}\left(\theta_{0}\right)$ is on the boundary of the ample cone, then it is in the interior of a facet since $\theta_{0}$ is assumed to be outside of any other walls and the map $L_{C}$ is submersive. It follows that $f$ is a primitive contraction in this case.

The type of a wall in the parameter space is defined as the type of the corresponding primitive birational contraction. If the morphism $f: \mathcal{M}_{\theta} \rightarrow$ $X_{\theta_{0}}$ is an isomorphism, then it is called a wall of type 0 . 


\section{Variations of moduli spaces}

Let $W_{R_{1}}$ be the wall separating $\theta$ and $\theta^{\prime}$, where $R_{1} \subset V$ is the support of a $\theta^{\prime}$-destabilizing submodule $S$ of a $\theta$-stable $\mathbb{C} \Gamma$-module $E$. Then $R_{2}:=V \backslash$ $R_{1}$ is the support of the quotient module $Q=E / S$ and one has $W_{R_{1}}=W_{R_{2}}$.

The unstable locus is the set

$$
Z=\left\{y \in \mathcal{M}_{\theta} \mid E_{y} \text { is not } \theta_{0} \text {-stable }\right\} \subset \mathcal{M}_{\theta}
$$

of strictly $\theta_{0}$-semistable representations, with the natural subscheme structure defined by setting the values of the arrows going out from $R_{1}$ to zero. Since the values of the arrows in the universal representation are given by square free monomials in local coordinates given in [IU08, Lemma 4.5], the subscheme $Z$ is reduced.

For a point $z \in Z$, the $\theta^{\prime}$-destabilizing sequence of the $\theta$-stable representation $E_{z}$ parametrized by $z$ is written as

$$
0 \rightarrow S_{z} \rightarrow E_{z} \rightarrow Q_{z} \rightarrow 0
$$

where $\operatorname{dim} S_{z}=\chi_{R_{1}}, \operatorname{dim} Q_{z}=\chi_{R_{2}}$ and $\theta\left(\chi_{R_{1}}\right)=-\theta\left(\chi_{R_{2}}\right)>0, \theta^{\prime}\left(\chi_{R_{1}}\right)=$ $-\theta^{\prime}\left(\chi_{R_{2}}\right)<0$.

Since $\theta_{0}$ is assumed to be generic on the wall, both $S_{z}$ and $Q_{z}$ are $\theta_{0}$-stable. The point $f(z) \in \mathcal{M}_{\theta_{0}}$ parametrizes the S-equivalence class of $\left[S_{z} \oplus Q_{z}\right]$.

Lemma 10.1 The fibers of the morphisms $f$ and $f^{\prime}$ in (6.2) at $f(z) \in Z_{0}$ are $\mathbb{P}\left(\operatorname{Ext}^{1}\left(Q_{z}, S_{z}\right)^{\vee}\right)$ and $\mathbb{P}\left(\operatorname{Ext}^{1}\left(S_{z}, Q_{z}\right)^{\vee}\right)$ respectively.

Proof. Recall that an S-equivalence class consists of objects with the same graded quotient with respect to the Harder-Narasimhan filtration. Any object in the S-equivalence class of $\left[S_{z} \oplus Q_{z}\right]$ must either be an extension of $Q_{z}$ by $S_{z}$ or an extension of $S_{z}$ by $Q_{z}$. The latter is $\theta$-unstable, so that any $\theta$-stable representation $E$ of $\Gamma$ in the same S-equivalence class as $\left[S_{z} \oplus Q_{z}\right]$ is obtained as a non-trivial extension of $Q_{z}$ by $S_{z}$;

$$
0 \rightarrow S_{z} \rightarrow E \rightarrow Q_{z} \rightarrow 0
$$

To show the converse, take any non-trivial extension $E^{\prime}$ of $Q_{z}$ by $S_{z}$ and assume that $E^{\prime}$ is not $\theta$-stable. Then the image of the destabilizing subobject $S^{\prime}$ of $E^{\prime}$ in the quotient $Q_{z}$ must be a non-zero subobject, since one would 
have $\theta\left(S^{\prime}\right)>0$ otherwise. This image destabilizes $Q_{z}$ with respect to the stability parameter $\theta_{0}$. This contradicts the $\theta_{0}$-stability of $Q_{z}$, so that any non-trivial extension $E^{\prime}$ of $Q_{z}$ by $S_{z}$ is $\theta$-stable. This shows that the fiber of $f$ at $f(z)$ is given by $\mathbb{P}\left(\operatorname{Ext}^{1}\left(Q_{z}, S_{z}\right)^{\vee}\right)$.

Similarly, $\theta^{\prime}$-stable representations of $\Gamma$ in the same S-equivalence class as $\left[S_{z} \oplus Q_{z}\right]$ are obtained as non-trivial extensions of $S_{z}$ by $Q_{z}$, and parametrized by $\mathbb{P}\left(\operatorname{Ext}^{1}\left(S_{z}, Q_{z}\right)^{\vee}\right)$.

Recall from Section 2 that the set $V$ of vertices is the set of connected components of the complement $T \backslash\left(\bigcup_{e \in E} e\right)$ of the union of edges of the dimer model. We identify $R_{1}$ and $R_{2}$ with the closures of $\bigcup_{v \in R_{1}} v$ and $\bigcup_{v \in R_{2}} v$ in $T$ respectively.

Lemma 10.2 $R_{1}$ and $R_{2}$ are connected.

Proof. Suppose $R_{1}=R_{1,1} \cup R_{1,2}$ is a disjoint union of two open subsets of $R_{1}$. Then $S_{z}$ is a direct sum of two submodules whose dimension vectors are $\chi_{R_{1,1}}$ and $\chi_{R_{1,2}}$ respectively. If both $R_{1,1}$ and $R_{1,2}$ are non-empty, then $S_{z}$ is the direct sum of two non-zero submodules, which cannot be $\theta_{0}$-stable. Hence $R_{1}$ is connected. The connectedness of $R_{2}$ is proved similarly.

Lemma 10.3 $S_{z}$ and $Q_{z}$ are simple; $\operatorname{End}\left(S_{z}\right)=\mathbb{C} \cdot \operatorname{id}_{S_{z}}$ and $\operatorname{End}\left(Q_{z}\right)=$ $\mathbb{C} \cdot \operatorname{id}_{Q_{z}}$.

Proof. This follows from the $\theta_{0}$-stability.

Lemma 10.4 If $z \in Z$ is in a two-dimensional $\mathbb{T}$-orbit, then $\operatorname{dim} \operatorname{Ext}^{1}\left(Q_{z}, S_{z}\right)$ is the number of connected components of the boundary $\partial R_{1}$ of $R_{1}$.

Proof. According to [IU08, Lemma 6.1], a point $z \in Z$ is in a twodimensional $\mathbb{T}$-orbit if and only if the arrows whose values are zero in the representation $E_{z}$ form a perfect matching. By using the $\mathbb{T}$-action, we may assume that all the non-zero values of arrows are 1 . Since $S_{z}$ is a submodule of $E_{z}$, one must have $\psi_{a}=0$ for any arrow $a$ from $R_{1}=\operatorname{Supp} S_{z}$ to $R_{2}=\operatorname{Supp} Q_{z}=V \backslash R_{1}$. This means that $R_{1}$ is a "sink" in the sense that any arrows going from $R_{1}$ is blocked by the perfect matching.

To give an extension

$$
0 \rightarrow S_{z} \rightarrow E \rightarrow Q_{z} \rightarrow 0
$$


of $Q_{z}$ by $S_{z}$ is the same thing as to assign numbers $\psi_{a} \in \mathbb{C}$ to each arrows $a$ from $R_{2}=\operatorname{Supp} Q$ to $R_{1}=\operatorname{Supp} S$. If two arrows $a$ and $b$ from $R_{2}$ to $R_{1}$ cross the same connected component of $\partial R_{1}$, then one must have $\psi_{a}=\psi_{b}$ by the relations of the quiver. If they cross different components, then the values are independent. Since $\operatorname{Ext}^{1}\left(Q_{z}, S_{z}\right)$ classifies extensions of the form (10.2), Lemma 10.4 is proved.

Lemma 10.5 There are no walls of type II.

Proof. Assume that $f: \mathcal{M}_{\theta} \rightarrow X_{\theta_{0}}$ contracts a surface to a point, and take a point $z$ in a two-dimensional $\mathbb{T}$-orbit on the contracted surface. Then for the $\theta^{\prime}$-destabilizing sequence

$$
0 \rightarrow S_{z} \rightarrow E_{z} \rightarrow Q_{z} \rightarrow 0
$$

one has $\operatorname{dim} \operatorname{Ext}^{1}\left(Q_{z}, S_{z}\right)=3$ by Lemma 10.1, so that the the boundary of $R_{1}$ has three connected components by Lemma 10.4. Lemma 10.2 shows that both $R_{1}$ and $R_{2}$ are connected. On the other hand, it is impossible to divide the torus $T$ into two connected pieces $R_{1}$ and $R_{2}$ in such a way that the boundary of $R_{1}$ has three connected components, and Lemma 10.5 is proved.

\section{Variations of tautological bundles}

Assume that the conditions $(\mathbf{T})+(\mathbf{E})$ hold for $\theta$, and consider the diagram (6.2). The resulting birational morphism $\mathcal{M}_{\theta} \rightarrow \mathcal{M}_{\theta^{\prime}}$ is either a flop or an isomorphism since both are crepant resolutions of $X_{\theta_{0}}$ by [IU08, Proposition 5.1]. In either cases, the strict transformation induces an isomorphism

$$
\operatorname{Pic} \mathcal{M}_{\theta} \stackrel{\sim}{\longrightarrow} \operatorname{Pic} \mathcal{M}_{\theta^{\prime}}
$$

of Picard groups. Let $\mathcal{L}_{v}$ and $\mathcal{L}_{v}^{\prime}$ be the tautological bundles on $\mathcal{M}_{\theta}$ and $\mathcal{M}_{\theta^{\prime}}$ respectively.

We prove the following in this section:

Theorem 11.1 There is an equivalence

$$
\Xi: D^{b} \operatorname{coh} \mathcal{M}_{\theta} \stackrel{\sim}{\longrightarrow} D^{b} \operatorname{coh} \mathcal{M}_{\theta^{\prime}}
$$


of the derived category such that $\Xi\left(\mathcal{L}_{v}\right)=\mathcal{L}_{v}^{\prime}$ for any $v \in V$.

This result is proved in [CI04] for McKay quivers by assuming the conditions $(\mathbf{T})+(\mathbf{E})$ for both $\theta$ and $\theta^{\prime}$. Here we work with general non-degenerate dimer models, and we assume the conditions $(\mathbf{T})+(\mathbf{E})$ only for $\theta$. Since there are only finitely many walls, Theorem 1.1 is an immediate consequence of Theorem 11.1.

\subsection{Type 0 case}

Assume that $f: \mathcal{M}_{\theta} \rightarrow X_{\theta_{0}}$ is an isomorphism, so that the diagram (6.2) induces an isomorphism $\phi: \mathcal{M}_{\theta} \stackrel{\sim}{\longrightarrow} \mathcal{M}_{\theta^{\prime}}$ of moduli spaces, and only tautological bundles change.

Recall from (10.1) that the unstable locus $Z$ is defined by setting the arrows going out from $R_{1}$ to zero. The zero locus of each arrow is a union of reduced toric divisors, and $Z$ is the intersection of such divisors.

Lemma 11.2 Every irreducible component of $Z$ is two-dimensional.

Proof. Recall that a family of representations of a quiver is given by a collection of line bundles and maps of the line bundles. Therefore, if two families of representations parametrized by a normal variety coincide in codimension one, then they must be the same family. Since $\mathcal{M}_{\theta} \backslash Z=\mathcal{M}_{\theta^{\prime}} \backslash Z$ parametrizes the same family and $Z$ is the locus where the two families are different, we are done.

Let $Z_{1}$ be a connected component of $Z$. Take $z \in Z_{1}$ and let

$$
0 \rightarrow S_{z} \rightarrow E_{z} \rightarrow Q_{z} \rightarrow 0
$$

be $\theta^{\prime}$-destabilizing sequence of $E_{z}$.

Lemma 11.3 Either $S_{z}$ or $Q_{z}$ is rigid for any $z \in Z_{1}$.

Proof. Since $f$ is of type 0 , one has $\operatorname{dim} \operatorname{Ext}^{1}\left(Q_{z}, S_{z}\right)=1$ by Lemma 10.1 . Assume $z$ is in a two-dimensional $\mathbb{T}$-orbit. Then Lemma 10.4 shows that $\partial R_{1}$ is connected. This implies that either $R_{1}$ or $R_{2}$ is simply connected. If $R_{1}$ is simply-connected, then the values $\psi_{a}$ for the representation $S_{z}$ can be normalized to 0 or 1 by gauge transformation, so that $S_{z}$ is rigid. If not, then $R_{2}$ is simply-connected and $Q_{z}$ is rigid.

Since $S_{z}$ and $Q_{z}$ are $\theta_{0}$-stable for any $z \in Z_{1}$, we have morphisms from $Z_{1}$ to the moduli spaces of $\theta_{0}$-stable representations with dimension vectors 
$\chi_{R_{1}}$ and $\chi_{R_{2}}$ respectively. A rigid representation forms a connected component of the moduli space and therefore if a rigid representation appears in a flat family of $\theta_{0}$-stable representations parametrized by the connected scheme $Z_{1}$, then it must be a constant family.

\subsubsection{Rigid subobject case}

We first assume that $S_{z}$ is rigid. This assumption implies that the family $\mathcal{S}$ of destabilizing submodules of the universal representation over $Z_{1}$ is constant. Let $S$ be the rigid destabilizing submodule of $E_{z}$ for some (and hence all) $z \in Z_{1}$.

Lemma 11.4 $\operatorname{Supp}\left(\Phi^{-1}(S)\right) \subset \mathcal{M}_{\theta}$ is compact.

Proof. Note that $\operatorname{Supp}\left(\Phi^{-1}(S)\right)$ is projective over the affine variety $X_{\theta}$. Therefore, if $\operatorname{Supp}\left(\Phi^{-1}(S)\right)$ is not compact, then $\operatorname{End}\left(\Phi^{-1}(S)\right)$ must be infinite-dimensional, which contradicts $\operatorname{End}\left(\Phi^{-1}(S)\right) \cong \operatorname{End}(S)=\mathbb{C} \cdot \operatorname{id}_{S}$ in Lemma 10.3.

Lemma $11.5 \chi\left(S, E_{y}\right)=0$ for any $y \in \mathcal{M}_{\theta}$.

Proof. Recall that $\chi\left(S, E_{y}\right)=\sum_{i=0}^{3}(-1)^{i} \operatorname{dim}_{\operatorname{Ext}^{i}}\left(S, E_{y}\right)$ is the Euler characteristic of $\mathbb{R} \operatorname{Hom}\left(S, E_{y}\right)$. Since $\operatorname{Supp}\left(\Phi^{-1}(S)\right)$ is compact, one can take $y \in \mathcal{M}_{\theta} \backslash \operatorname{Supp}\left(\Phi^{-1}(S)\right)$, which clearly satisfies $\chi\left(\Phi^{-1}(S), \mathcal{O}_{y}\right)=0$. Since skyscraper sheaves are numerically equivalent, this shows $\chi\left(S, E_{y}\right)=$ $\chi\left(\Phi^{-1}(S), \mathcal{O}_{y}\right)=0$ for any $y \in \mathcal{M}_{\theta}$.

Lemma 11.6 For any $y \in \mathcal{M}_{\theta} \backslash Z$ and any $i \in \mathbb{Z}$, one has $\operatorname{Ext}^{i}\left(S, E_{y}\right)=0$.

Proof. Since both $S$ and $E_{y}$ are $\mathbb{C} \Gamma$-modules, the Calabi-Yau property of $D^{b} \operatorname{coh} \mathcal{M}_{\theta} \cong D^{b} \bmod \mathbb{C} \Gamma$ implies $\operatorname{Ext}^{i}\left(S, E_{y}\right)=0$ for $i \neq 0,1,2,3$. For $i=0$, one has $\operatorname{Hom}\left(S, E_{y}\right)=0$ if $y \notin Z$, since the image of $S$ in $E_{y}$ will be a $\theta^{\prime}$-destabilizing submodule otherwise. For $i=3$, one has $\operatorname{Ext}^{3}\left(S, E_{y}\right) \cong$ $\operatorname{Hom}\left(E_{y}, S\right)^{\vee}=0$, since the kernel of an element in $\operatorname{Hom}\left(E_{y}, S\right)$ will be a $\theta$-destabilizing submodule of $E_{y}$. For $i=2$ and a point $z \in Z_{1}$, consider the Jordan-Hölder filtration

$$
0 \rightarrow S \rightarrow E_{z} \rightarrow Q_{z} \rightarrow 0
$$

of $E_{z}$. The assumption $y \notin Z$ implies $\operatorname{Hom}\left(E_{y}, Q_{z}\right)=0$. Since $\Phi$ is an equivalence, we have $\operatorname{Ext}^{1}\left(E_{y}, E_{z}\right) \cong \operatorname{Ext}^{1}\left(\mathcal{O}_{y}, \mathcal{O}_{z}\right)=0$, which implies 
$\operatorname{Ext}^{1}\left(E_{y}, S\right)=0$. The remaining case $i=1$ follows from $\chi\left(S, E_{y}\right)=0$ shown in Lemma 11.5.

Lemma 11.7 One has $\operatorname{Supp} \Phi^{-1}(S)=Z_{1}$.

Proof. One has $\operatorname{Hom}^{i}\left(\Phi^{-1}(S), \mathcal{O}_{y}\right)=0$ for any $y \notin Z$ and any $i \in \mathbb{Z}$, which implies $\operatorname{Supp} \Phi^{-1}(S) \subset Z$. On the other hand, one also has $\operatorname{Hom}\left(S, E_{y}\right) \cong \mathbb{C}$ if $y \in Z_{1}$, so that Supp $\Phi^{-1}(S) \supset Z_{1}$. Indecomposability of $S$ implies that of $\Phi^{-1}(S)$, so that the support of $\Phi^{-1}(S)$ is connected. Since $Z_{1}$ is a connected component, one obtains $\operatorname{Supp} \Phi^{-1}(S)=Z_{1}$.

Lemma $11.8 \quad Z_{1}$ is compact.

Proof. This is clear from Lemma 11.4 and Lemma 11.7.

Lemma 11.9 $Z$ is connected.

Proof. Assume for contradiction that there is another connected component $Z_{2}$ of $Z$. First we consider the case when $Z_{2}$ parametrizes representations whose destabilizing subobject $S^{\prime}$ is rigid. The classes $[S]$ and $\left[S^{\prime}\right]$ in the Grothendieck group $K(\Gamma)$ are the same, since both of them are in the kernel of a stability parameter $\theta_{0}$ which is generic on the wall. This contradicts the fact that the classes of $\Phi^{-1}(S)$ and $\Phi^{-1}\left(S^{\prime}\right)$ in $K\left(\mathcal{M}_{\theta}\right)$ must be linearly independent, since their supports are unions of compact toric divisors which are mutually disjoint.

Next we consider the case when $Z_{2}$ parametrizes representations containing a rigid quotient $Q$. The support of $\Phi^{-1}(Q)$ coincides with $Z_{2}$ as in the subrepresentation case. On the other hand, the sum $[S]+[Q]$ of the classes of $S$ and $Q$ in $K(\Gamma)$ is zero. This contradicts the linear independence of $\left[\Phi^{-1}(S)\right]$ and $\left[\Phi^{-1}(Q)\right]$ in $K\left(\mathcal{M}_{\theta}\right)$, obtained in just the same way as above.

Lemma 11.10 $Z$ is compact.

Proof. This is an immediate consequence of Lemma 11.8 and Lemma 11.9.

Lemma 11.11 For any $z \in Z$, one has

$$
\operatorname{Hom}^{i}\left(\Phi^{-1}(S), \mathcal{O}_{z}\right)= \begin{cases}\mathbb{C} & i=0,1 \\ 0 & \text { otherwise }\end{cases}
$$


Proof. For $i=0$, one has $\operatorname{Hom}\left(S, E_{z}\right) \cong \mathbb{C}$ for $z \in Z$. For $i=3$, one has $\operatorname{Ext}^{3}\left(S, E_{z}\right)=\operatorname{Hom}\left(E_{z}, S\right)=0$ for any $z \in \mathcal{M}_{\theta}$ by $\theta$-stability of $E_{z}$. For $i=2$, consider the $\theta^{\prime}$-destabilizing sequence

$$
0 \rightarrow S \rightarrow E_{z} \rightarrow Q_{z} \rightarrow 0 .
$$

One has $\operatorname{dim} \operatorname{Ext}^{1}\left(Q_{z}, S\right)=1$ by Lemma 10.1, and rigidity of $S$ implies $\operatorname{dim} \operatorname{Ext}^{1}(S, S)=0$. Now the long exact sequence of $\operatorname{Ext}^{*}(-, S)$ associated with (11.1) shows $\operatorname{Ext}^{1}\left(E_{z}, S\right)=0$. The remaining case $i=1$ follows from $\chi\left(\Phi^{-1}(S), \mathcal{O}_{z}\right)=0$ just as in Lemma 11.6.

Lemma $11.12 \quad \Phi^{-1}(S)$ is a line bundle on a Cartier divisor whose reduced part is $Z$.

Proof. Lemma 11.11 shows that the cohomology sheaves $\mathcal{H}^{i}\left(\Phi^{-1}(S)\right)$ is non-zero only if $i=0$ or -1 , and both of them are supported on $Z$. Then by [BM02, Proposition 5.4], the object $\Phi^{-1}(S)$ has homological dimension at most one, i.e., the object $\Phi^{-1}(S)$ is quasi-isomorphic to a complex of the form

$$
0 \rightarrow \mathcal{P}^{-1} \rightarrow \mathcal{P}^{0} \rightarrow 0
$$

where $\mathcal{P}^{i}$ are locally-free sheaves. Since the support of $\Phi^{-1}(S)$ is a proper subset, the locally-free sheaves $\mathcal{P}^{-1}$ and $\mathcal{P}^{0}$ have the same rank and $\Phi^{-1}(S)$ is isomorphic to an $\mathcal{O}_{\mathcal{M}_{\theta}}$-module. The condition $\operatorname{Hom}\left(\Phi^{-1}(S), \mathcal{O}_{z}\right) \cong \mathbb{C}$ for $z \in Z$ implies that locally near $z$, one can take a surjection $\mathcal{Q}^{0} \rightarrow \Phi^{-1}(S)$ from a line bundle $\mathcal{Q}^{0}$. The kernel $\mathcal{Q}^{-1}$ of this surjection is a line bundle since $\Phi^{-1}(S)$ has homological dimension one. It follows that $\Phi^{-1}(S)$ is locally isomorphic to the cokernel of a morphism of line bundles, and Lemma 11.12 is proved.

Lemma $11.13 \Phi^{-1}(S)$ is a line bundle on $Z$.

Proof. Lemma 11.12 shows that $\Phi^{-1}(S)$ is a sheaf of $\mathcal{O}_{n Z}$-modules for sufficiently large $n$. On the other hand, the sum $\omega$ of all the small cycles lies in the center of $\mathbb{C} \Gamma$ and acts on $S$ as zero. It follows from the explicit description of local coordinates of $\mathcal{M}_{\theta}$ around torus fixed points given in [IU08, Section 4] that the zero locus of $\omega$ as a function on $\mathcal{M}_{\theta}$ is the union of all the toric divisors with multiplicity one. This shows that $\Phi^{-1}(S)$ in 
fact is a sheaf of $\mathcal{O}_{Z}$-modules.

Lemma 11.14 For any vertex $v \in R_{1}$, one has an isomorphism

$$
\left.\Phi^{-1}(S) \stackrel{\sim}{\longrightarrow} \mathcal{L}_{v}^{\vee}\right|_{Z}
$$

of line bundles on $Z$.

Proof. Rigidity of $S$ implies that any non-zero arrow between two vertices in $R_{1}$ does not vanish on $Z$, so that the restrictions $\left.\mathcal{L}_{w}\right|_{Z}$ for $w \in R_{1}$ are mutually isomorphic. It follows that $\left.\left(\mathcal{L}_{v}^{\vee} \otimes \mathcal{L}_{w}\right)\right|_{Z} \cong \mathcal{O}_{Z}$ for any $w \in R_{1}$, and one has $S=H^{0}\left(\left.\bigoplus_{w \in R_{1}}\left(\mathcal{L}_{w} \otimes \mathcal{L}_{v}^{\vee}\right)\right|_{Z}\right)$. Note that $\bigoplus_{w \in V} \mathcal{L}_{w} \otimes \mathcal{L}_{v}^{\vee}$ is the universal family, which is normalized in such away that the line bundle corresponding to the vertex $v$ is trivial, and $S \otimes_{\mathbb{C}} \mathcal{O}_{Z}$ is the (trivial) family of destabilizing subobjects of the restriction of this universal family to $Z$.

It follows from the definition of the functor $\Phi$ that

$$
\begin{aligned}
S & =H^{0}\left(\left.\left(\bigoplus_{w \in R_{1}} \mathcal{L}_{w}\right) \otimes \mathcal{L}_{v}^{\vee}\right|_{Z}\right) \subset H^{0}\left(\left.\left(\bigoplus_{w \in V} \mathcal{L}_{w}\right) \otimes \mathcal{L}_{v}^{\vee}\right|_{Z}\right) \\
& \cong \mathcal{H}^{0}\left(\Phi\left(\left.\mathcal{L}_{v}^{\vee}\right|_{Z}\right)\right)
\end{aligned}
$$

where $\mathcal{H}^{0}$ denotes the 0 -th cohomology functor with respect to the standard $t$-structure on $D^{b} \bmod \mathbb{C} \Gamma$. Since $\mathcal{H}^{i}\left(\Phi\left(\left.\mathcal{L}_{v}^{\vee}\right|_{Z}\right)\right)=0$ for $i<0$, the above inclusion induces a morphism

$$
\alpha: S \rightarrow \Phi\left(\left.\mathcal{L}_{v}^{\vee}\right|_{Z}\right)
$$

such that the composition $S \rightarrow \Phi\left(\left.\mathcal{L}_{v}^{\vee}\right|_{Z}\right) \rightarrow \Phi\left(\left.\mathcal{L}_{v}^{\vee}\right|_{z}\right) \cong E_{z}$ is non-zero for any $z \in Z$. Then $\Phi^{-1}(\alpha)$ is a morphism $\left.\Phi^{-1}(S) \rightarrow \mathcal{L}_{v}^{\vee}\right|_{Z}$ such that the composition $\left.\left.\Phi^{-1}(S) \rightarrow \mathcal{L}_{v}^{\vee}\right|_{Z} \rightarrow \mathcal{L}_{v}^{\vee}\right|_{z}$ is non-zero for any $z \in Z$. Since they are both line bundles on $Z$, the morphism $\Phi^{-1}(\alpha)$ must be an isomorphism.

Corollary 11.15 For any $v \in R_{1}$, one has $\Phi\left(\left.\mathcal{L}_{v}^{\vee}\right|_{Z}\right) \cong S$.

Set $\mathcal{F}=\left.\mathcal{L}_{v}(Z)\right|_{Z}$, so that we have an exact sequence

$$
0 \rightarrow \mathcal{L}_{v} \rightarrow \mathcal{L}_{v}(Z) \rightarrow \mathcal{F} \rightarrow 0
$$


The dual of the short exact sequence

$$
\left.0 \rightarrow \mathcal{L}_{v}^{\vee}(-Z) \rightarrow \mathcal{L}_{v}^{\vee} \rightarrow \mathcal{L}_{v}^{\vee}\right|_{Z} \rightarrow 0
$$

gives the distinguished triangle

$$
\left(\left.\mathcal{L}_{v}^{\vee}\right|_{Z}\right)^{\vee} \rightarrow \mathcal{L}_{v} \rightarrow \mathcal{L}_{v}(Z) \rightarrow\left(\left.\mathcal{L}_{v}^{\vee}\right|_{Z}\right)^{\vee}[1]
$$

which shows that $\mathcal{F} \cong\left(\left.\mathcal{L}_{v}^{\vee}\right|_{Z}\right)^{\vee}[1] \cong \Phi^{-1}(S)^{\vee}[1]$.

Corollary 11.16 One has

$$
\mathbb{R} \operatorname{Hom}\left(\mathcal{F}, \mathcal{L}_{w}\right)= \begin{cases}\mathbb{C}[-1] & w \in R_{1}, \\ 0 & w \in R_{2} .\end{cases}
$$

Proof. (11.2) and Corollary 11.15 imply

$$
\mathbb{R} \Gamma\left(\left(\left.\mathcal{L}_{v}^{\vee}\right|_{Z}\right) \otimes \mathcal{L}_{w}\right)= \begin{cases}\mathbb{C} & w \in R_{1}, \\ 0 & w \in R_{2},\end{cases}
$$

which together with $\mathcal{F} \cong\left(\left.\mathcal{L}_{v}^{\vee}\right|_{Z}\right)^{\vee}[1]$ gives the assertion.

Lemma 11.17 Let $\bigoplus_{w \in V} \mathcal{L}_{w}^{\prime \prime}$ be the kernel of the morphism from $\bigoplus_{w \in V} \mathcal{L}_{w}$ to the family $\mathcal{Q}$ of quotients parametrized by $Z$;

$$
\bigoplus_{w \in V} \mathcal{L}_{w}^{\prime \prime}=\operatorname{Ker}\left(\bigoplus_{w \in V} \mathcal{L}_{w} \rightarrow \mathcal{Q}\right)
$$

Then the tautological bundle $\bigoplus_{w \in V} \mathcal{L}_{w}^{\prime}$ on $\mathcal{M}_{\theta^{\prime}}$ is given by

$$
\bigoplus_{w \in V} \mathcal{L}_{w}^{\prime}= \begin{cases}\bigoplus_{w \in V} \mathcal{L}_{w}^{\prime \prime} & v_{0} \in R_{1}, \\ \bigoplus_{w \in V} \mathcal{L}_{w}^{\prime \prime}(Z) & v_{0} \in R_{2}\end{cases}
$$

where $v_{0}$ is the fixed vertex such that $\mathcal{L}_{v_{0}} \cong \mathcal{L}_{v_{0}}^{\prime}$ is the trivial line bundle as in Section 3.

Proof. Consider the following diagram: 


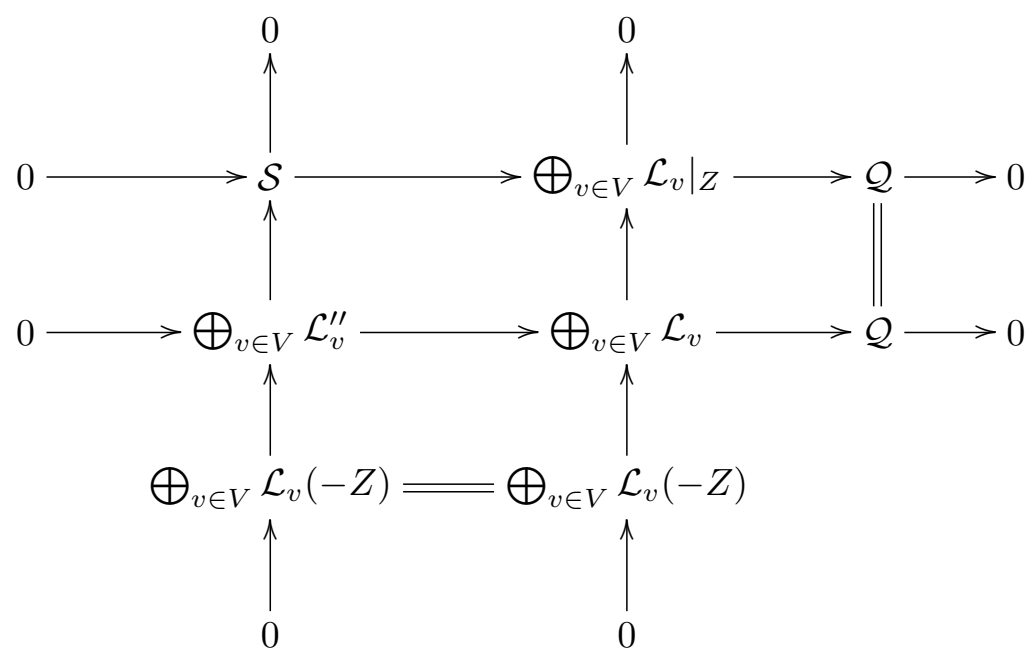

Here, the row

$$
\left.0 \rightarrow \mathcal{S} \rightarrow \bigoplus_{v \in V} \mathcal{L}_{v}\right|_{Z} \rightarrow \mathcal{Q} \rightarrow 0
$$

is the family of $\theta^{\prime}$-destabilizing sequences on $Z$. The diagram (11.5) gives the following diagram:

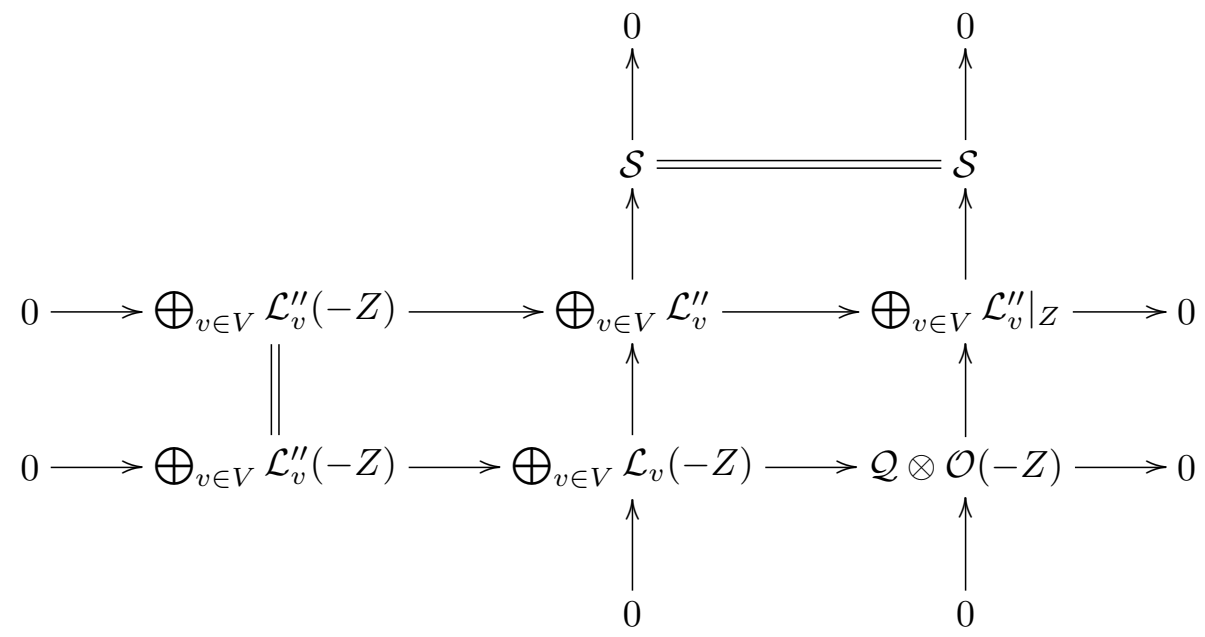


Here, the middle row of (11.6) describes the restriction of $\bigoplus_{v} \mathcal{L}_{v}^{\prime \prime}$ to $Z$, and the bottom row comes from the middle row of (11.5) by tensoring with $\mathcal{O}(-Z)$. The middle column of (11.6) comes from the left column of (11.5), and the right column of (11.6) is induced from the other parts of (11.6).

The column

$$
\left.0 \rightarrow \mathcal{Q} \otimes \mathcal{O}(-Z) \rightarrow \bigoplus_{v \in V} \mathcal{L}_{v}^{\prime \prime}\right|_{Z} \rightarrow \mathcal{S} \rightarrow 0
$$

gives a family of $\theta$-destabilizing sequences on $Z$. We show that for every $z \in Z$, the extension

$$
\left.\left.0 \rightarrow \mathcal{Q} \otimes \mathcal{O}(-Z)\right|_{z} \rightarrow \bigoplus_{v \in V} \mathcal{L}_{v}^{\prime \prime}\right|_{z} \rightarrow \mathcal{S}_{z} \rightarrow 0
$$

is non-split.

Recall that $Z$ is defined by the system of equations corresponding to the arrows whose sources are in $R_{1}$ and whose targets are in $R_{2}$. In terms of local coordinates in [IU08], these equations are represented by monomials. Since $Z$ is a Cartier divisor, $Z$ is locally defined by one of these monomials. Thus, there is an arrow $a$ with $s(a) \in R_{1}, t(a) \in R_{2}$ and an open neighborhood $U$ of $z$ such that $\left.\left.\mathcal{L}_{t(a)}\right|_{U} \cong \mathcal{L}_{s(a)}(Z)\right|_{U}$ where the map $\mathcal{L}_{s(a)} \rightarrow \mathcal{L}_{t(a)}$ over $U$ is identified with the natural inclusion map $\left.\left.\mathcal{L}_{s(a)}\right|_{U} \rightarrow \mathcal{L}_{s(a)}(Z)\right|_{U}$. This shows that $\left.\mathcal{L}_{s(a)}^{\prime \prime}\right|_{U} \rightarrow \mathcal{L}_{t(a)}^{\prime \prime} \mid U$ is an isomorphism, proving (11.7) is non-split.

The non-splitting of (11.7) shows that $\left.\bigoplus_{v \in V} \mathcal{L}_{v}^{\prime \prime}\right|_{z}$ is actually $\theta^{\prime}$-stable. Thus $\bigoplus_{v \in V} \mathcal{L}_{v}^{\prime \prime}$ is a family of $\theta^{\prime}$-stable representations parametrized by $\mathcal{M}_{\theta}$. Then there is a morphism $\mathcal{M}_{\theta} \rightarrow \mathcal{M}_{\theta^{\prime}}$ which is the identity outside $Z$. Therefore this morphism is the identity and the tautological bundles $\bigoplus_{v \in V} \mathcal{L}_{v}^{\prime}$ and $\bigoplus_{v \in V} \mathcal{L}_{v}^{\prime \prime}$ coincide up to a line bundle. Since we normalized the tautological bundles so that $\mathcal{L}_{v_{0}}$ and $\mathcal{L}_{v_{0}}^{\prime}$ are trivial line bundles, we obtain (11.4).

Definition 11.18 ([ST01, Definitions 1.1 and 2.5]) An object $\mathcal{G}$ in a triangulated category with the trivial Serre functor of degree 3 is a spherical object if

$$
\operatorname{Ext}^{k}(\mathcal{G}, \mathcal{G})= \begin{cases}\mathbb{C} & k=0,3 \\ 0 & \text { otherwise }\end{cases}
$$


For a spherical object $\mathcal{G}$, the spherical twist functor defined by

$$
T_{\mathcal{G}}=\text { Cone }\{\operatorname{hom}(\mathcal{G},-) \otimes \mathcal{G} \stackrel{\mathrm{ev}}{\longrightarrow}-\}: D^{b} \operatorname{coh} \mathcal{M}_{\theta} \rightarrow D^{b} \operatorname{coh} \mathcal{M}_{\theta}
$$

A spherical twist is an autoequivalence of triangulated categories [ST01, Proposition 2.10].

Lemma 11.19 $\mathcal{F}$ is a spherical object.

Proof. Lemma 10.3 gives

$$
\operatorname{Hom}(\mathcal{F}, \mathcal{F}) \cong \operatorname{Ext}^{3}(\mathcal{F}, \mathcal{F})^{\vee} \cong \mathbb{C}
$$

and the rigidity of $S$ gives

$$
\operatorname{Ext}^{1}(\mathcal{F}, \mathcal{F}) \cong \operatorname{Ext}^{2}(\mathcal{F}, \mathcal{F})^{\vee} \cong 0
$$

Proposition 11.20 One has $T_{\mathcal{F}}\left(\bigoplus_{w \in V} \mathcal{L}_{w}\right) \cong \begin{cases}\bigoplus_{w \in V} \mathcal{L}_{w}^{\prime}(Z) & v_{0} \in R_{1}, \\ \bigoplus_{w \in V} \mathcal{L}_{w}^{\prime} & v_{0} \in R_{2}\end{cases}$

Proof. Corollary 11.16 shows that

$$
T_{\mathcal{F}}\left(\mathcal{L}_{w}\right) \cong \begin{cases}\text { Cone }\left\{\mathcal{F}[-1] \rightarrow \mathcal{L}_{w}\right\} & w \in R_{1} \\ \mathcal{L}_{w} & w \in R_{2}\end{cases}
$$

If $w \in R_{1}$, then one has $\mathcal{F}=\left.\mathcal{L}_{w}(Z)\right|_{Z}$, and the exact sequence

$$
\left.0 \rightarrow \mathcal{L}_{w} \rightarrow \mathcal{L}_{w}(Z) \rightarrow \mathcal{L}_{w}(Z)\right|_{Z} \rightarrow 0
$$

shows that $T_{\mathcal{F}}\left(\mathcal{L}_{w}\right) \cong \mathcal{L}_{w}(Z)$. Since

$$
\begin{aligned}
\bigoplus_{w \in V} \mathcal{L}_{w}^{\prime \prime} & =\operatorname{Ker}\left(\bigoplus_{w \in V} \mathcal{L}_{w} \rightarrow \mathcal{Q}\right) \\
& = \begin{cases}\mathcal{L}_{w} & w \in R_{1} \\
\mathcal{L}_{w}(-Z) & w \in R_{2}\end{cases}
\end{aligned}
$$

by (11.3), one has $T_{\mathcal{F}}\left(\mathcal{L}_{w}\right)=\mathcal{L}_{w}^{\prime \prime}(Z)$ for both $w \in R_{1}$ and $w \in R_{2}$; 


$$
T_{\mathcal{F}}\left(\mathcal{L}_{w}\right) \cong \bigoplus_{w \in V} \mathcal{L}_{w}^{\prime \prime}(Z)
$$

This, together with (11.4), immediately implies Proposition 11.20.

Proposition 11.20 shows Theorem 11.1 with either $\Xi=T_{\mathcal{F}}$ or $\Xi=$ $\mathcal{O}(-Z) \otimes T_{\mathcal{F}}$ in the type 0 case with rigid subobject.

\subsubsection{Rigid quotient case}

Assume that the quotient object $Q_{z}$ instead of the subobject $S_{z}$ is rigid. The discussion in the rigid subobject case can be adapted to this case by replacing $\operatorname{Ext}^{i}\left(S, E_{y}\right)$ with $\operatorname{Ext}^{i}\left(E_{y}, Q\right)$, and one shows that $Z$ is a connected Cartier divisor and

$$
\Phi^{-1}(Q) \cong \mathcal{L}_{v}^{\vee} \otimes \omega_{Z}[2]
$$

for $v \in R_{2}$ where $\omega_{Z}$ is the dualizing sheaf of $Z$ and [2] is the 2-shift in the derived category. The object $\mathcal{F}=\left.\mathcal{L}_{v}\right|_{Z}$ is a spherical object and Theorem 11.1 holds with $\Xi=T_{\mathcal{F}}^{-1}$ or $\Xi=\mathcal{O}(Z) \otimes T_{\mathcal{F}}^{-1}$.

\subsection{Type I case}

Assume that the wall $W=W_{R_{1}}$ separating the chambers $C$ and $C^{\prime}$ is of type I. Let $\ell \subset \mathcal{M}_{\theta}$ be the curve contracted by $f$.

Lemma 11.21 One has $\theta\left(\varphi_{C}\left(\mathcal{O}_{\ell}\right)\right)>0$ for any $\theta \in C$ and $\theta_{0}\left(\varphi_{C}\left(\mathcal{O}_{\ell}\right)\right)=0$ for any $\theta_{0} \in W$.

Proof. For $\theta \in C$, one has $\theta\left(\varphi_{C}\left(\mathcal{O}_{\ell}\right)\right)=\left.\operatorname{deg} L_{C}(\theta)\right|_{\ell}$ by (9.6), which is positive since $L_{C}(\theta) \in \operatorname{Pic}\left(\mathcal{M}_{\theta}\right)_{\mathbb{Q}}$ is ample.

For $\theta_{0} \in W$, the $\mathbb{Q}$-line bundle $L_{C}\left(\theta_{0}\right)$ is the pull-back of an ample $\mathbb{Q}$ line bundle in $\operatorname{Pic}\left(\overline{\mathcal{M}}_{\theta_{0}}\right)_{\mathbb{Q}}$, so that $\left.\operatorname{deg} L_{C}\left(\theta_{0}\right)\right|_{\ell}$ cannot be positive since $\ell$ is contracted by the morphism $\mathcal{M}_{\theta} \rightarrow \overline{\mathcal{M}}_{\theta_{0}}$.

Lemma 11.22 If $v_{0} \in R_{2}$, then $\left.\operatorname{deg} \mathcal{L}_{v}\right|_{\ell}$ is either 0 or 1 for any $v \in V$. If $v_{0} \in R_{1}$, then $\left.\operatorname{deg} \mathcal{L}_{v}\right|_{\ell}$ is either 0 or -1 for any $v \in V$.

Proof. One of the defining inequalities for the chamber $C$ is given by $\theta\left(\chi_{R_{1}}\right)>0$, which is also written as $\theta\left(\varphi_{C}\left(\mathcal{O}_{\ell}\right)\right)>0$ by Lemma 11.21. This implies the existence of a positive rational number $q$ satisfying $\theta\left(\varphi_{C}\left(\mathcal{O}_{\ell}\right)\right)=$ $q \theta\left(\chi_{R_{1}}\right)$ for any $\theta \in \Theta$. Since $\Theta$ is defined as $\Theta=\left\{\theta \in \operatorname{Hom}\left(\mathbb{Z}^{V}, \mathbb{Z}\right) \mid\right.$ $\left.\theta\left(\chi_{V}\right)=0\right\}$, the fact that $\theta\left(q \chi_{R_{1}}-\varphi_{C}\left(\mathcal{O}_{\ell}\right)\right)=0$ for any $\theta \in \Theta$ implies the 
existence of $k \in \mathbb{Q}$ such that $q \chi_{R_{1}}-\varphi_{C}\left(\mathcal{O}_{\ell}\right)=k_{V}$. By substituting any $v_{2} \in R_{2}$ to this equality, one shows that $k=-\varphi_{C}\left(\mathcal{O}_{\ell}\right)\left(v_{2}\right)$ is an integer. Since $\varphi_{C}([\mathrm{pt}])=\chi_{V}$, one has $\varphi_{C}\left(\mathcal{O}_{\ell}(k)\right)=q \chi_{R_{1}}$. Since $q$ is positive and $\mathcal{O}_{\ell}(k)$ is not divisible in $K_{0}\left(\mathcal{M}_{\theta}\right)$, one has $q=1$, so that $\varphi_{C}\left(\mathcal{O}_{\ell}(k)\right)=\chi_{R_{1}}$. Since

$$
\varphi_{C}\left(\mathcal{O}_{\ell}(k)\right)(v)=\sum_{i}(-1)^{i} \operatorname{dim} H^{i}\left(\mathcal{L}_{v} \otimes \mathcal{O}_{\ell}(k)\right)
$$

by the definition of $\varphi_{C}$, one has

$$
\operatorname{deg}\left(\mathcal{L}_{v} \otimes \mathcal{O}_{\ell}(k)\right)= \begin{cases}0 & v \in R_{1} \\ -1 & v \in R_{2}\end{cases}
$$

Since $\mathcal{L}_{v_{0}}$ is the trivial bundle by our normalization, one concludes that

$$
k=\operatorname{deg}\left(\mathcal{L}_{v_{0}} \otimes \mathcal{O}_{\ell}(k)\right)= \begin{cases}0 & v_{0} \in R_{1}, \\ -1 & v_{0} \in R_{2} .\end{cases}
$$

Let $Z \subset \mathcal{M}_{\theta}$ be the $\theta^{\prime}$-unstable locus.

Lemma $\mathbf{1 1 . 2 3} \ell$ is a connected component of $Z$.

Proof. Since $\operatorname{deg}\left(\mathcal{L}_{v} \otimes \mathcal{O}_{\ell}(k)\right)$ is 0 or -1 , the object

$$
S=\Phi_{C}\left(\mathcal{O}_{\ell}(k)\right)=\mathbb{R} \Gamma\left(\bigoplus_{v \in V} \mathcal{L}_{v} \otimes \mathcal{O}_{\ell}(k)\right)
$$

in the derived category $D^{b} \bmod \mathbb{C} \Gamma$ is in fact an object of $\bmod \mathbb{C} \Gamma$, which is rigid since $\mathcal{O}_{\ell}(k)$ is rigid. The equivalence $\Phi_{C}$ gives $\operatorname{Hom}_{\mathcal{O}_{\mathcal{M}_{\theta}}}\left(\mathcal{O}_{\ell}(k), \mathcal{O}_{y}\right) \cong$ $\operatorname{Hom}_{\Gamma}\left(S, E_{y}\right)$, so that $S$ destabilizes $E_{y}$ if and only if $y$ is on the rational curve $\ell$.

Since $\theta_{0}$ is a generic stability parameter on the wall $W_{R_{1}}$, the destabilizing submodule $S_{y}$ for $y \in Z$ is a $\theta_{0}$-stable representation with dimension vector $\chi_{R_{1}}$. This gives a morphism from $Z$ to the moduli space of $\theta_{0}$-stable representations with dimension vector $\chi_{R_{1}}$. Rigidity of $S$ implies that $[S]$ is an isolated point in this moduli space, so that $\ell$ is a connected component of $Z$. 


\section{Proposition $\mathbf{1 1 . 2 4}$ One has $Z=\ell$.}

Proof. Let $Z_{c}=Z \backslash \ell$ be the complement of $\ell$ in $Z$, and assume for contradiction that $Z_{c}$ is non-empty. Take a connected component $Z_{1}$ of $Z_{c}$. The same argument as in the type 0 case shows that $Z_{1}$ is a compact Cartier divisor and one has either $\Phi_{C}\left(\left.\mathcal{L}_{v}^{\vee}\right|_{Z_{1}}\right)=S$ or $\Phi_{C}\left(\mathcal{L}_{v}^{\vee} \otimes \omega_{Z_{1}}\right)=Q$, so that $\varphi_{C}\left(\left.\mathcal{L}_{v}^{\vee}\right|_{Z_{1}}\right)=\chi_{R_{1}}$ or $\varphi_{C}\left(\mathcal{L}_{v}^{\vee} \otimes \omega_{Z_{1}}\right)=\chi_{R_{2}}$. This contradicts $\varphi_{C}\left(\mathcal{O}_{\ell}(k)\right)$ $=\chi_{R_{1}}$ and $\varphi_{C}\left(\mathcal{O}_{\ell}(k-1)\right)=-\chi_{R_{2}}$, and Proposition 11.24 is proved.

Corollary 11.25 For any $v \in V$, the tautological bundle $\mathcal{L}_{v}^{\prime}$ on $\mathcal{M}_{\theta^{\prime}}$ is the strict transform of the tautological bundle $\mathcal{L}_{v}$ on $\mathcal{M}_{\theta}$.

Proof. Since the tautological bundle does not change outside of the unstable locus, the fact that the unstable locus $Z=\ell$ is of codimension two implies that the tautological bundle on $\mathcal{M}_{\theta^{\prime}}$ is the strict transform of the tautological bundle on $\mathcal{M}_{\theta}$.

Lemma 11.26 The diagram (6.2) is the Atiyah flop.

Proof. Corollary 11.25 and (4.2) show that the strict transform of $\mathcal{O}_{\mathcal{M}_{\theta}}(1) \otimes \mathcal{O}_{X_{\theta_{0}}}(1)^{\vee}$ is $\mathcal{O}_{\mathcal{M}_{\theta^{\prime}}}(-1) \otimes \mathcal{O}_{X_{\theta_{0}}}(1)$. Since $f$ is a small primitive contraction of a toric Calabi-Yau 3-fold, the diagram (6.2) is the Atiyah flop.

Let $\widetilde{\mathcal{M}}=\mathcal{M}_{\theta} \times_{X_{\theta_{0}}} \mathcal{M}_{\theta^{\prime}}$ be the fiber product of $\mathcal{M}_{\theta}$ and $\mathcal{M}_{\theta^{\prime}}$ over $X_{\theta_{0}}$. The natural projections will be denoted by $p: \widetilde{\mathcal{M}} \rightarrow \mathcal{M}_{\theta}$ and $q: \widetilde{\mathcal{M}} \rightarrow \mathcal{M}_{\theta^{\prime}}$. Both $p$ and $q$ are blow-ups along the unstable loci, and the exceptional set $E$ is a divisor in $\widetilde{\mathcal{M}}$ isomorphic to $\mathbb{P}^{1} \times \mathbb{P}^{1}$.

Theorem 11.27 (Bondal and Orlov [BO]) The functor

$$
\mathbb{R} q_{*} \circ \mathbb{L} p^{*}: D^{b} \operatorname{coh} \mathcal{M}_{\theta} \rightarrow D^{b} \operatorname{coh} \mathcal{M}_{\theta^{\prime}}
$$

is an equivalence of triangulated categories, whose inverse is given by

$$
\mathbb{R} p_{*}\left(\mathcal{O}_{\widetilde{\mathcal{M}}}(E) \otimes \mathbb{L} p^{*}(-)\right): D^{b} \operatorname{coh} \mathcal{M}_{\theta^{\prime}} \rightarrow D^{b} \operatorname{coh} \mathcal{M}_{\theta}
$$

Lemma $\mathbf{1 1 . 2 8}$ If $v_{0} \in R_{2}$, then one has

$$
\mathbb{R} q_{*} \circ \mathbb{L} p^{*}\left(\mathcal{L}_{v}\right) \cong \mathcal{L}_{v}^{\prime}
$$


for any $v \in V$. If $v_{0} \in R_{1}$, then one has

$$
\mathbb{R} q_{*}\left(\mathcal{O}_{\widetilde{\mathcal{M}}}(E) \otimes \mathbb{L} p^{*}\left(\mathcal{L}_{v}\right)\right) \cong \mathcal{L}_{v}^{\prime}
$$

for any $v \in V$.

Proof. If $v_{0} \in R_{2}$, then $\operatorname{deg} \mathcal{L}_{v}$ is either 0 or 1 for any $v \in V$ by Lemma 11.22 , and $\mathbb{R} q_{*} \circ \mathbb{L} p^{*}\left(\mathcal{L}_{v}\right)=q_{*}\left(p^{*} \mathcal{L}_{v}\right)$ is the strict transform of $\mathcal{L}_{v}$.

If $v_{0} \in R_{1}$, then $\operatorname{deg} \mathcal{L}_{v}$ is either 0 or -1 for any $v \in V$ and $\mathbb{R} q_{*}\left(\mathcal{O}_{\widetilde{\mathcal{M}}}(E) \otimes\right.$ $\left.\mathbb{L} p^{*}\left(\mathcal{L}_{v}\right)\right)=q_{*}\left(\mathcal{O}_{\widetilde{\mathcal{M}}}(E) \otimes p^{*} \mathcal{L}_{v}\right)$ is the strict transform of $\mathcal{L}_{v}$.

This shows Theorem 11.1 for type I with $\Xi=\mathbb{R} q_{*} \circ \mathbb{L} p^{*}$ or $\mathbb{R} q_{*}\left(\mathcal{O}_{\widetilde{\mathcal{M}}}(E) \otimes\right.$ $\left.\mathbb{L} p^{*}(-)\right)$.

\subsection{Type III case}

Assume that the wall $W$ separating the chambers $C$ and $C^{\prime}$ is of type III, so that $f: \mathcal{M}_{\theta} \rightarrow X_{\theta_{0}}$ contracts a toric divisor $D \subset \mathcal{M}_{\theta}$ to a torus-invariant curve $B \subset X_{\theta_{0}}$.

Lemma 11.29 The morphism $f^{\prime}: \mathcal{M}_{\theta^{\prime}} \rightarrow X_{\theta_{0}}$ also contracts a divisor $D^{\prime} \subset \mathcal{M}_{\theta^{\prime}}$ to the curve $B \subset X_{\theta_{0}}$, and the birational map $\mathcal{M}_{\theta} \rightarrow \mathcal{M}_{\theta^{\prime}}$ associated with the diagram (6.2) extends to an isomorphism.

Proof. This is because $\mathcal{M}_{\theta}$ and $\mathcal{M}_{\theta^{\prime}}$ are obtained as the blow-up of $X_{\theta_{0}}$ along the curve $B$ just as in [CI04, 6.2].

Let $\ell$ be a curve in $D$ which contracts to a point in $B$.

Lemma 11.30 One has $\theta\left(\varphi_{C}\left(\mathcal{O}_{\ell}\right)\right)>0$ for any $\theta \in C$ and $\theta_{0}\left(\varphi_{C}\left(\mathcal{O}_{\ell}\right)\right)=0$ for any $\theta_{0} \in W$.

Proof. The proof is identical to that of Lemma 11.21

Proposition 11.31 One has $Z=D$.

Proof. One can prove that $S=\Phi_{C}\left(\mathcal{O}_{\ell}(k)\right)$ is an object of the abelian category just as in the type I case, where $k=-1$ if $v_{0} \in R_{2}$, and $k=0$ if $v_{0} \in R_{1}$. The object $S$ is a $\theta^{\prime}$-destabilizing subobject of $E_{z}$ for $z \in \ell$. Unlike the type I case, the object $S$ is not rigid, and $B$ is a connected component of the moduli space of $\theta_{0}$-stable representations with dimension vector $\chi_{R_{1}}$, so that $D$ is a connected component of $Z$. The complement $Z \backslash D$ is empty just as in the type I case. 
Lemma 11.32 If $v_{0} \in R_{1}$, then one has

$$
\mathcal{L}_{v}^{\prime}= \begin{cases}\mathcal{L}_{v}(-D) & \left.\operatorname{deg} \mathcal{L}_{v}\right|_{\ell}=-1 \\ \mathcal{L}_{v} & \left.\operatorname{deg} \mathcal{L}_{v}\right|_{\ell}=0\end{cases}
$$

If $v_{0} \in R_{2}$, then one has

$$
\mathcal{L}_{v}^{\prime}= \begin{cases}\mathcal{L}_{v} & \left.\operatorname{deg} \mathcal{L}_{v}\right|_{\ell}=0 \\ \mathcal{L}_{v}(D) & \left.\operatorname{deg} \mathcal{L}_{v}\right|_{\ell}=1\end{cases}
$$

Proof. This is proved in the same way as in Lemma 11.17.

Let $\widetilde{\mathcal{M}}=\mathcal{M}_{\theta} \times_{X_{\theta_{0}}} \mathcal{M}_{\theta^{\prime}}$ be the fiber product of $\mathcal{M}_{\theta}$ and $\mathcal{M}_{\theta^{\prime}}$ over $X_{\theta_{0}}$. The natural projections will be denoted by $p: \widetilde{\mathcal{M}} \rightarrow \mathcal{M}_{\theta}$ and $q: \widetilde{\mathcal{M}} \rightarrow \mathcal{M}_{\theta^{\prime}}$. Let $\omega_{\widetilde{\mathcal{M}}}$ be the dualizing sheaf of $\widetilde{\mathcal{M}}$. The scheme $\widetilde{\mathcal{M}}$ has two irreducible components; one is isomorphic to $\mathcal{M}:=\mathcal{M}_{\theta} \cong \mathcal{M}_{\theta^{\prime}}$ and the other is $D \times_{B} D$. One has exact sequences

$$
0 \rightarrow \mathcal{O}_{\mathcal{M}}(-D) \rightarrow \mathcal{O}_{\widetilde{\mathcal{M}}} \rightarrow \mathcal{O}_{D \times{ }_{B} D} \rightarrow 0
$$

and

$$
0 \rightarrow \omega_{\mathcal{M}} \rightarrow \omega_{\widetilde{\mathcal{M}}} \rightarrow \omega_{D \times_{B} D}\left(\Delta_{D}\right) \rightarrow 0
$$

where $\Delta_{D} \subset D \times_{B} D$ is the diagonal.

Lemma 11.33 If $v_{0} \in R_{1}$, then one has

$$
\mathbb{R} q_{*} \circ \mathbb{L} p^{*}\left(\mathcal{L}_{v}\right) \cong \mathcal{L}_{v}^{\prime}
$$

for any $v \in V$. If $v_{0} \in R_{2}$, then one has

$$
\mathbb{R} q_{*}\left(\omega_{\widetilde{\mathcal{M}}} \otimes \mathbb{L} p^{*}\left(\mathcal{L}_{v}\right)\right) \cong \mathcal{L}_{v}^{\prime}
$$

for any $v \in V$.

Proof. This is proved by using Lemma 11.32, (11.9) and (11.10).

Theorem 11.34 The functor

$$
\mathbb{R} q_{*} \circ \mathbb{L} p^{*}: D^{b} \operatorname{coh} \mathcal{M}_{\theta} \rightarrow D^{b} \operatorname{coh} \mathcal{M}_{\theta^{\prime}}
$$


is an equivalence of triangulated categories, whose inverse is given by

$$
\mathbb{R} p_{*}\left(\omega_{\widetilde{\mathcal{M}}} \otimes \mathbb{L} p^{*}(-)\right): D^{b} \operatorname{coh} \mathcal{M}_{\theta^{\prime}} \rightarrow D^{b} \operatorname{coh} \mathcal{M}_{\theta} .
$$

Proof. These functors are compositions of Horja's EZ-transforms [Hor05] and the tensor products by line bundles as explained in [CI04, Remark 7.6].

This shows Theorem 11.1 for type $\mathbb{I I I}$ with $\Xi=\mathbb{R} q_{*} \circ \mathbb{L} p^{*}$ or $\mathbb{R} p_{*}\left(\omega_{\widetilde{\mathcal{M}}} \otimes\right.$ $\left.\mathbb{L} p^{*}(-)\right)$.

\section{Projective crepant resolutions as moduli spaces}

In this section, we give a proof of Corollary 1.2. Since the proof is completely parallel to that of the main theorem of [CI04], we will be brief here and refer the reader to [CI04] for details.

Let $Y=\mathcal{M}_{\theta}$ be the moduli space for some generic stability parameter $\theta \in \Theta$, and $X=X_{0}$ be the normalization of the irreducible component of the moduli space $\overline{\mathcal{M}}_{0}$ for the stability parameter $0 \in \Theta$ containing the torus $\mathbb{T}$. According to [IU08, Proposition 6.3], the space $X$ is the Gorenstein affine toric variety associated with the characteristic polygon of the dimer model $G$. Since $Y$ is a toric variety, its ample cone $\operatorname{Amp}(Y)$ is an interior of a convex rational polyhedral cone. Since a flop is an isomorphism in codimension one, one can identify the Picard groups of any crepant resolutions. The union

$$
\overline{\operatorname{Mov}(Y)}=\bigcup_{Y^{\prime}} \overline{\operatorname{Amp}\left(Y^{\prime}\right)} \subset \operatorname{Pic}(Y)_{\mathbb{Q}}
$$

of the nef cones over the set of projective crepant resolutions $Y^{\prime}$ is the closure of the movable cone. Ample cones of birational Calabi-Yau 3-folds give a polyhedral decomposition of the movable cone [Kaw88].

Consider the diagram

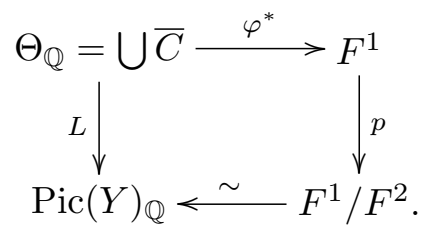


The space $\Theta_{\mathbb{Q}}$ is divided into chambers by walls of types 0 , I, and III. The map $L: \Theta_{\mathbb{Q}} \rightarrow \operatorname{Pic}(Y)_{\mathbb{Q}}$ is a piecewise-linear map which is given by $L_{C}$ on each chamber $C \subset \Theta_{\mathbb{Q}}$. The map $\varphi^{*}$ is defined similarly by the collection $\varphi_{C}^{*}: C \rightarrow F^{1}$ of linear maps. The image of $L$ sits inside the movable cone $\operatorname{Mov}(Y)$, and one asks whether $L$ surjects onto $\operatorname{Mov}(Y)$. Lemma 11.26 shows that the moduli space flops across a wall $W \subset \Theta_{\mathbb{Q}}$ of type I. Since any crepant resolution $Y^{\prime} \rightarrow X$ is related to $Y$ by a sequence of flops, it suffices to show the following to prove Corollary 1.2:

Proposition 12.1 Let $Y=\mathcal{M}_{\theta}$ be the moduli space for a generic stability parameter $\theta \in \Theta$. For any flopping wall $\bar{W} \subset \operatorname{Pic}(Y)_{\mathbb{Q}}$ on the boundary of the nef cone $\overline{\operatorname{Amp}(Y)}$, there is a chamber $C \subset \Theta$ and a wall $W$ of type $I$ of the chamber $C$ such that $L_{C}(W)=\bar{W}$.

Although the chamber $C$ may not be the chamber $C_{\theta}$ containing the stability parameter $\theta$, one can reach the chamber containing the desired wall $W$ of type $I$ on the boundary by crossing a finite number of walls of type 0 . To show this, it is natural to consider the union $\bigcup_{\mathcal{M}_{C} \cong Y} \varphi_{C}^{*}(\bar{C})$ of closures $\bar{C}$ of chambers such that $\mathcal{M}_{C}$ is isomorphic to $Y$, where $\mathcal{M}_{C}$ is the moduli space $\mathcal{M}_{\theta}$ for one (and hence any) stability parameter $\theta \in C$.

Unfortunately, this is still not enough, since adjacent chambers in $\Theta$ may not be sent to adjacent cones in $F^{1}$. This comes from the ambiguity in the choice of the tautological bundle, which we have fixed by hand by choosing a vertex $v_{0} \in V$ and setting $\mathcal{L}_{v_{0}}=\mathcal{O}_{\mathcal{M}_{\theta}}$. To solve this problem, [CI04] considers the union $\bigcup_{L \in \operatorname{Pic}^{c}(Y)} \bigcup_{\mathcal{M}_{C} \cong Y} L \otimes \varphi_{C}^{*}(\bar{C})$ over the subgroup $\operatorname{Pic}^{c}(Y)$ of $\operatorname{Pic}(Y)$ generated by $\mathcal{O}_{Y}(S)$ for compact divisors $S$. The reason that it suffices to consider only compact divisors comes from the fact that the unstable locus for a type 0 wall is a compact divisor, so that the variation of the tautological bundle given in Lemma 11.17 is described solely in terms of a compact divisor. Let $\operatorname{Amp}^{\prime}(Y) \subset \overline{\operatorname{Amp}(Y)}$ be the complement of the walls of type II. The advantage of working with $\operatorname{Pic}^{c}(Y)$ instead of $\operatorname{Pic}(Y)$ lies in the following lemma:

Lemma 12.2 ([CI04, Lemma 8.1]) Let $S_{1}, \ldots, S_{b}$ be a basis of $\operatorname{Pic}^{c}(Y)$. If $p(\xi) \in \operatorname{Amp}^{\prime}(Y)$ for $\xi \in F^{1}$, then $\left[\mathcal{O}_{S_{1}} \otimes \xi\right], \ldots,\left[\mathcal{O}_{S_{b}} \otimes \xi\right]$ are linearly independent elements in $F^{2}$.

To prove Proposition 12.1, one uses the following description of the chamber $C$ : 
Theorem 12.3 ([CI04, Theorem 5.9]) One has $\theta \in C$ if and only if

- for every exceptional curve $\ell$, one has $\theta\left(\varphi_{C}\left(\mathcal{O}_{\ell}\right)\right)>0$, and

- for every compact reduced divisor $D$ and every vertex $v \in V$, one has

$$
\theta\left(\varphi_{C}\left(\mathcal{L}_{v}^{\vee} \otimes \omega_{D}\right)\right)<0 \quad \text { and } \quad \theta\left(\varphi_{C}\left(\left.\mathcal{L}_{v}^{\vee}\right|_{D}\right)\right)>0 .
$$

Sketch of proof. The inequality arising from a wall of type I or III is of the form $\theta\left(\varphi_{C}\left(\mathcal{O}_{\ell}\right)\right)>0$ by Lemma 11.21 and Lemma 11.30. The inequality coming from a wall of type 0 is of the form $\theta\left(\varphi_{C}\left(\left.\mathcal{L}_{v}^{\vee}\right|_{D}\right)\right)>0$ in the case when the unstable locus $D$ parameterizes rigid submodules by Corollary 11.15 and $\theta(\operatorname{dim} S)>0$, and of the form $\theta\left(\varphi_{C}\left(\mathcal{L}_{v}^{\vee} \otimes \omega_{D}\right)\right)<0$ in the case when $D$ parameterizes rigid quotients by $(11.8)$ and $\theta(\operatorname{dim} Q)<0$. Conversely, any parameter $\theta$ in $C$ has to satisfy the inequalities (12.1) for any compact reduced divisor by the same argument as in the proof of [CI04, Lemma 5.7].

The following proposition concludes the proof of Proposition 12.1:

Proposition 12.4 ([CI04, Proposition 8.2]) For every $\xi \in p^{-1}\left(\mathrm{Amp}^{\prime}(Y)\right)$, there is a neighborhood $N(\xi)$ of $\xi$ in $F^{1}$ such that only finitely-many pairs $(L, C)$ of $L \in \operatorname{Pic}^{c}(Y)$ and chamber $C \subset \Theta$ satisfy $N(\xi) \cap L \otimes \varphi_{C}^{*}(\bar{C}) \neq \emptyset$. Moreover, one has

$$
p^{-1}\left(\operatorname{Amp}^{\prime}(Y)\right) \subset \bigcup_{L \in \operatorname{Pic}^{c}(Y)} \bigcup_{\mathcal{M}_{C} \cong Y} L \otimes \varphi_{C}^{*}(\bar{C}) .
$$

Sketch of proof. The first statement is a local finiteness, which comes from Lemma 12.2 and Theorem 12.3; Theorem 12.3 shows that $\varphi_{C}^{*}(C)$ is bounded in the fiber direction of $p$, and Lemma 12.2 shows that taking the union over $\operatorname{Pic}^{c}(Y)$ does not destroy the local finiteness.

The second statement essentially comes from the fact that the derived equivalence caused by crossing a wall of type 0 is a spherical twist, which does not change the orientation in the 3 -dimensional case. It follows that if a chamber $C^{\prime}$ is on the other side of the wall $W$ from the chamber $C$, then $\varphi_{C^{\prime}}^{*}\left(C^{\prime}\right)$ is on the other side of $\varphi_{C}^{*}(W)$ from $\varphi_{C}(C)$ up to tensor by a line bundle $L \in \operatorname{Pic}^{c}(Y)$ associated with a compact divisor.

The detail of the proof is identical to the one in [CI04], and we omit it here. 
Proposition 12.1 is an immediate consequence of Proposition 12.4; the second statement ensures that for any wall $\bar{W} \subset \operatorname{Pic}(Y)_{\mathbb{Q}}$ of type $\mathrm{I}$, one can go arbitrarily close to that wall by moving in the space of stability parameters and tensoring line bundles $L \in \operatorname{Pic}^{c}(Y)$. The local finiteness in the first statement ensures that one in fact can find finitely many walls of type 0 such that by crossing them, one can reach the desired wall.

Example 12.5 Consider the dimer model in Figure 12.1, which is discussed in [BM, Section 5.1]. The space of stability parameters

$$
\Theta_{\mathbb{R}}=\left\{\left(\theta_{1}, \theta_{2}, \theta_{3}\right) \in \mathbb{R}^{3} \mid \theta_{1}+\theta_{2}+\theta_{3}=0\right\}
$$

is two-dimensional, and there are six chambers in $\Theta_{\mathbb{R}}$ as shown in Figure 12.2. The walls on the line $\theta_{2}+\theta_{3}=0$ are of type $\mathrm{III}$, and the other walls are of type I. There are no walls of type 0 since there are no compact exceptional surfaces.

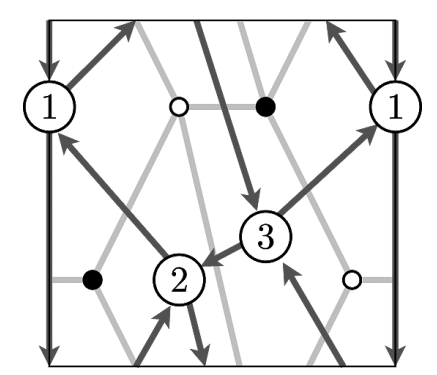

Figure 12.1. A dimer model.

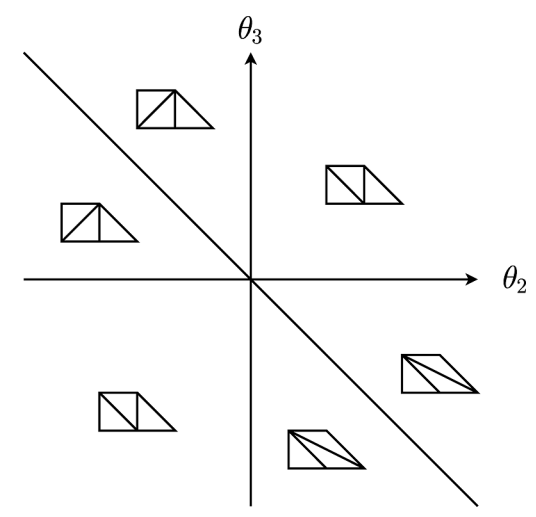

Figure 12.2. Walls and chambers.

Example 12.6 We give an example in which a single flop is not achieved by a single wall crossing as in [CI04, Example 9.13]. Consider the dimer model in Figure 12.3, where $\Theta_{\mathbb{R}}$ is 4-dimensional. First consider the stability parameter $\left(\theta_{1}, \theta_{2}, \theta_{3}, \theta_{4}, \theta_{5}\right)=(-4,1,1,1,1)$. The representations of $\Gamma$ corresponding to the $\mathbb{T}$-fixed points of $\mathcal{M}_{\theta}$ are shown in Figures 12.6-12.10, where only non-zero arrows are drawn and the edges corresponding to zero arrows are painted black. Note that $\theta$-stability is equivalent to the condition that every vertex is reachable from the vertex 1 through non-zero arrows. 


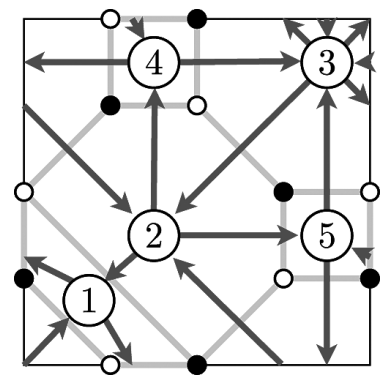

Figure 12.3. A dimer model.

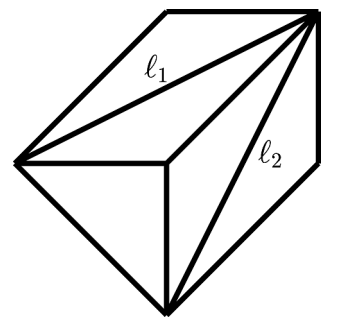

Figure 12.4. The fan of $\mathcal{M}_{\theta}$.

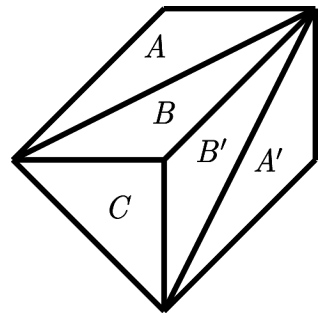

Figure 12.5.

$\mathbb{T}$-fixed points.

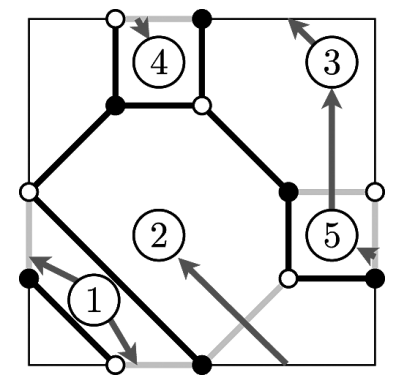

Figure 12.8. $B$.

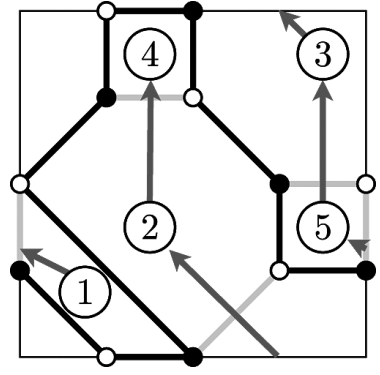

Figure 12.6. A.

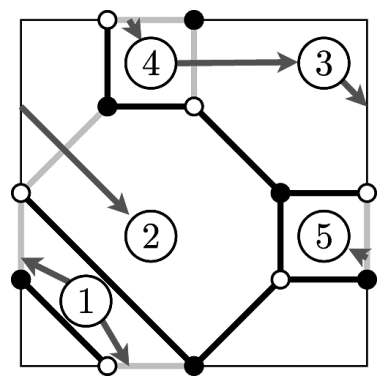

Figure 12.9. $B^{\prime}$.

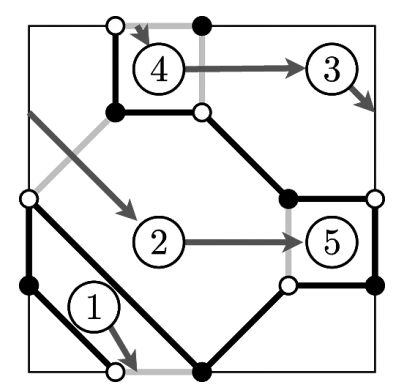

Figure 12.7. $A^{\prime}$.

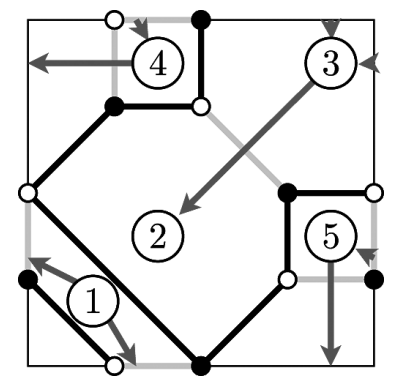

Figure 12.10. $C$.

We can check that the chamber containing $\theta$ is defined by the inequalities

$$
\begin{aligned}
\theta_{2} & >0, \\
\theta_{2}+\theta_{3} & >0, \\
\theta_{4} & >0, \\
\theta_{5} & >0 .
\end{aligned}
$$


The first two inequalities correspond to walls of type 0 and the others correspond to walls of type I, which induce flops along the curves $\ell_{1}$ and $\ell_{2}$ respectively.

We can flop twice to obtain the crepant resolution in Figure 12.11 by crossing two walls.

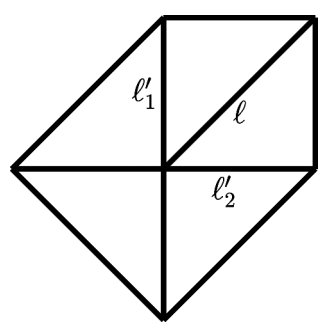

Figure 12.11. The fan of $\mathcal{M}_{C}$.

The corresponding chamber $C \subset \Theta_{\mathbb{R}}$ is defined by the inequalities

$$
\begin{aligned}
\theta_{2} & >0, \\
\theta_{2}+\theta_{3}+\theta_{4}+\theta_{5} & >0, \\
\theta_{4} & <0, \\
\theta_{5} & <0 .
\end{aligned}
$$

The walls defined by $\theta_{2}=0$ and $\theta_{2}+\theta_{3}+\theta_{4}+\theta_{5}=0$ are of type 0. The walls defined by $\theta_{4}=0$ and $\theta_{5}=0$ are of type I, which correspond to the flops along the curves $\ell_{1}^{\prime}$ and $\ell_{2}^{\prime}$ respectively. This shows that there is no wall which induces the flop along $\ell$. The adjacent chamber $C^{\prime}$ separated by the wall $\theta_{2}=0$ of type 0 is defined by the inequalities

$$
\begin{aligned}
\theta_{2} & <0, \\
\theta_{3} & >0, \\
\theta_{2}+\theta_{3}+\theta_{4}+\theta_{5} & >0, \\
\theta_{2}+\theta_{4} & <0, \\
\theta_{2}+\theta_{5} & <0 .
\end{aligned}
$$

$\mathcal{M}_{C^{\prime}}$ is isomorphic to $\mathcal{M}_{C}$ but now the wall $\theta_{2}+\theta_{3}+\theta_{4}+\theta_{5}=0$ is a wall 
of type I which induces the flop along $\ell$.

Thus the essential feature of [CI04, Example 9.13] can be observed in a smaller example in the case of dimer models. In fact, the pentagon in Figure 12.4 can be regarded as a part of the triangle in [CI04, Example 9.13].

Acknowledgment We thank the anonymous referee for suggestion a number of improvements. A. I. is supported by Grant-in-Aid for Scientific Research (No. 18540034). K. U. is supported by Grant-in-Aid for Young Scientists (No. 24740043).

\section{References}

[BKR01] Bridgeland T., King A. and Reid M., The McKay correspondence as an equivalence of derived categories. J. Amer. Math. Soc. 14(3) (2001), 535-554 (electronic).

[BM] Bender M. and Mozgovoy S., Crepant resolutions and brane tilings II: Tilting bundles, arXiv:0909.2013.

[BM02] Bridgeland T. and Maciocia A., Fourier-Mukai transforms for K3 and elliptic fibrations. J. Algebraic Geom. 11(4) (2002), 629-657.

[BO] Bondal A. and Orlov D., Semiorthogonal decomposition for algebraic varieties, arXiv:alg-geom/9506012.

[Bon89] Bondal A. I., Representations of associative algebras and coherent sheaves. Izv. Akad. Nauk SSSR Ser. Mat. 53(1) (1989), 25-44.

[CI04] Craw A. and Ishii A., Flops of G-Hilb and equivalences of derived categories by variation of GIT quotient. Duke Math. J. 124(2) (2004), 259-307.

[DH98] Dolgachev I. V. and Hu Y., Variation of geometric invariant theory quotients. Inst. Hautes Études Sci. Publ. Math. (1998), no. 87, 5-56, With an appendix by Nicolas Ressayre.

[Ful93] Fulton W., Introduction to toric varieties, Annals ofMathematics Studies, vol. 131, Princeton University Press, Princeton, NJ, 1993, The William H. Roever Lectures in Geometry.

[Hor05] Horja R. P., Derived category automorphisms from mirror symmetry. Duke Math. J. 127(1) (2005), 1-34.

[HVdB07] Hille L. and Van den Bergh M., Fourier-Mukai transforms, Handbook of tilting theory, London Math. Soc. Lecture Note Ser., vol. 332, Cambridge Univ. Press, Cambridge, 2007, pp. 147-177.

[IU] Ishii A. and Ueda K., Dimer models and the special McKay correspondence. arXiv:0905.0059. 
[IU08] Ishii A. and Ueda K., On moduli spaces of quiver representations associated with dimer models, Higher dimensional algebraic varieties and vector bundles, RIMS Kôkyûroku Bessatsu, B9, Res. Inst. Math. Sci. (RIMS), Kyoto, 2008, pp. 127-141.

[Kaw88] Kawamata Y., Crepant blowing-up of 3-dimensional canonical singularities and its application to degenerations of surfaces. Ann. of Math. (2) $\mathbf{1 2 7}(1)$ (1988), 93-163.

[Ken07] Kennaway K. D., Brane tilings. Internat. J. Modern Phys. A 22(18) (2007), 2977-3038.

[Kin94] King A. D., Moduli of representations of finite-dimensional algebras. Quart. J. Math. Oxford Ser. (2) 45(180) (1994), 515-530.

[Ric89] Rickard J., Morita theory for derived categories. J. London Math. Soc. (2) 39(3) (1989), 436-456.

[ST01] Seidel P. and Thomas R., Braid group actions on derived categories of coherent sheaves. Duke Math. J. 108(1) (2001), 37-108.

[Tha96] Thaddeus M., Geometric invariant theory and flips. J. Amer. Math. Soc. 9(3) (1996), 691-723.

[TU10] Toda Y. and Uehara H., Tilting generators via ample line bundles. Adv. Math. 223(1) (2010), 1-29.

[vdB04] van den Bergh M., Non-commutative crepant resolutions, The legacy of Niels Henrik Abel, Springer, Berlin, 2004, pp. 749-770.

[Wil92] Wilson P. M. H., The Kähler cone on Calabi-Yau threefolds. Invent. Math. 107(3) (1992), 561-583.

Akira IsHII

Division of Mathematical and Information Sciences Hiroshima University

1-7-1 Kagamiyama, Higashi-Hiroshima, 739-8521, Japan

E-mail: akira141@hiroshima-u.ac.jp

Kazushi UEDA

Graduate School of Mathematical Sciences

The University of Tokyo

1-8-1 Komaba, Meguro-ku, Tokyo 153-8914, Japan

E-mail: kazushi@ms.u-tokyo.ac.jp 